Dossier Temas de fonología otomangue

\title{
Fonotáctica consonántica y rasgos laríngeos de las lenguas mazatecas
}

Consonant phonotactics and laryngeal features in Mazatec languages

Mario E. Chávez Peón

Israel Filio García

Centro de Investigaciones y Estudios Superiores en Antropología Social mariochavezpeon@ciesas.edu.mx realfilio@gmail.com

Original recibido: 2020/08/28

Dictamen enviado al autor: 2020/11/03

Aceptado: 2021/08/23

\begin{abstract}
The present work describes and analyzes all the possible segmental sequences within the Mazatec languages at the level of the syllable. The basic template is $\mathrm{CV}$, although in native words a complex onset of fricative and plosive is possible (consonant clusters of [+continuant][-continuant] segments). This simple syllabic structure is maintained despite a high level of laryngeal complexity, realized as secondary features. It is this analysis that determines to a large degree the possible syllabic structures of these systems. This research is based on a large corpus and linguistic documentation resulting from the Nanginá Project. We depart from the proposal that the Mazatec linguistic group consists of 10 languages, or intelligible zones, and 20 linguistic variants. This dialect proposal is illustrated with a large number of examples
\end{abstract}

Cómo citar: Chávez Peón, Mario E. \& Filio García, Israel. 2021. Fonotáctica consonántica y rasgos laríngeos de las lenguas mazatecas. Cuadernos de Lingüística de El Colegio de México 8, e238. Dor: 10.24201/clecm.v8i0.238. 
throughout this work, showing possible consonant clusters and examples of each zone.

Keywords: Mazatec; phonology; consonant clusters; laryngeal features

\section{Resumen}

El presente trabajo describe y analiza todas las secuencias segmentales posibles dentro de las lenguas mazatecas a nivel de sílaba. El patrón básico es $\mathrm{CV}$, aunque en palabras nativas se permite un inicio complejo de fricativa más plosiva (secuencias consonánticas de segmentos [+continuo][-continuo]). Esta estructura silábica simple se mantiene a pesar de su notable complejidad laríngea realizada como rasgos secundarios. Este análisis determina en gran medida las posibles estructuras silábicas de estos sistemas. Esta investigación se sustenta en un amplio corpus y documentación lingüística fruto del Proyecto Nanginá. Partimos de la propuesta de que la agrupación lingüística mazateca consta de 10 lenguas, o zonas inteligibles, y 20 variantes lingüísticas. Esta propuesta dialectal se ilustra con una gran cantidad de ejemplos a lo largo del trabajo, sus posibles secuencias consonánticas y ejemplos de cada zona.

Palabras clave: mazateco; fonología; secuencias consonánticas; rasgos laríngeos

\section{INTRODUCCIÓN}

La fonotáctica se refiere a las secuencias o combinaciones posibles de los fonemas en una lengua, así como sus restricciones. Este estudio permite entender buena parte de la distribución de los sonidos de 
un sistema determinado y trazar un puente entre el inventario fonológico y la secuencia hablada de una lengua en la formación de unidades prosódicas como la sílaba y la palabra (ver Uchihara 2021, en este mismo volumen/dossier).

La estructura silábica prototípica de las lenguas mazatecas cuenta con un inicio obligatorio y un núcleo silábico, es decir una sílaba $\mathrm{CV}$, que incluye un gran número de rasgos secundarios posibles. Los ataques o inicio complejos muestran altas restricciones, lo que solamente permite la secuencia de fricativa más plosiva (se utilizará este término para incluir oclusivas y africadas), salvo en préstamos relativamente recientes. Asimismo, no se permiten las codas o consonantes a final de sílaba, por lo tanto, tampoco existen secuencias heterosilábicas de consonantes.

Así, como parte del entramado (morfo)fonológico de las lenguas mazatecas, el objetivo de este trabajo es ofrecer una descripción y análisis de su fonotáctica para arrojar luz sobre sus restricciones, la organización de sus rasgos, así como sobre diferentes estratos fonológicos en sus sistemas. Como marco teórico nos serviremos de diferentes análisis fonotácticos y silábicos como Pike \& Pike (1947), Blevins (1995; cf. Blevins 2003), Morelli (1999) y Zec (2007) entre otros, así como la teoría de rasgos distintivos (Jakobson, Fant \& Halle 1952; Chomsky \& Halle 1968), particularmente Hall (2007). De manera esquemática, implementaremos también la teoría de optimidad (Prince \& Smolensky (1993 [2004]); Kager 1999) para enunciar las restricciones que dan cuenta de la fonotáctica mazateca. Lo anterior, no solo implica abonar a la descripción de una de las 68 agrupaciones 
lingüísticas originarias del territorio mexicano, sino también delinear metodologías y análisis teóricos que pueden implementarse a otras agrupaciones y enriquecer, particularmente, nuestro conocimiento fonológico de las lenguas otomangues.

Después de la presente introducción, en $\S 2$ ofrecemos un panorama de las lenguas y variantes mazatecas, así como de los trabajos previos que se han realizado sobre ellas a nivel fonológico. Posteriormente, en $\S 3$ describimos la diversidad segmental de estas lenguas en los inicios simples, con ejemplos de todas las variantes, organizados por clases naturales según los rasgos que las componen. En $\S 4$ detallamos los inicios complejos y las restricciones globales de la fonotáctica $\mathrm{y}$, finalmente, en $\S 5$, presentamos las conclusiones.

\section{AnteCEDENTES: LENGUAS MAZATECAS y ANÁlisis PREVIOS}

En 2008, el Instituto Nacional de Lenguas Indígenas, a través del Catálogo de Lenguas Indígenas Nacionales (CLIN, INALI 2008), incluyó a la agrupación lingüística mazateca como uno de los 68 grupos etnolingüísticos de México y propuso 16 variantes para esta agrupación. El Proyecto Nanginá de Documentación Lingüística y Dialectología Mazateca (Chávez Peón 2018) retomó esas bases con el propósito de registrar de manera detallada toda la variación geolingüística del Pueblo mazateco (ver Figura 1). 


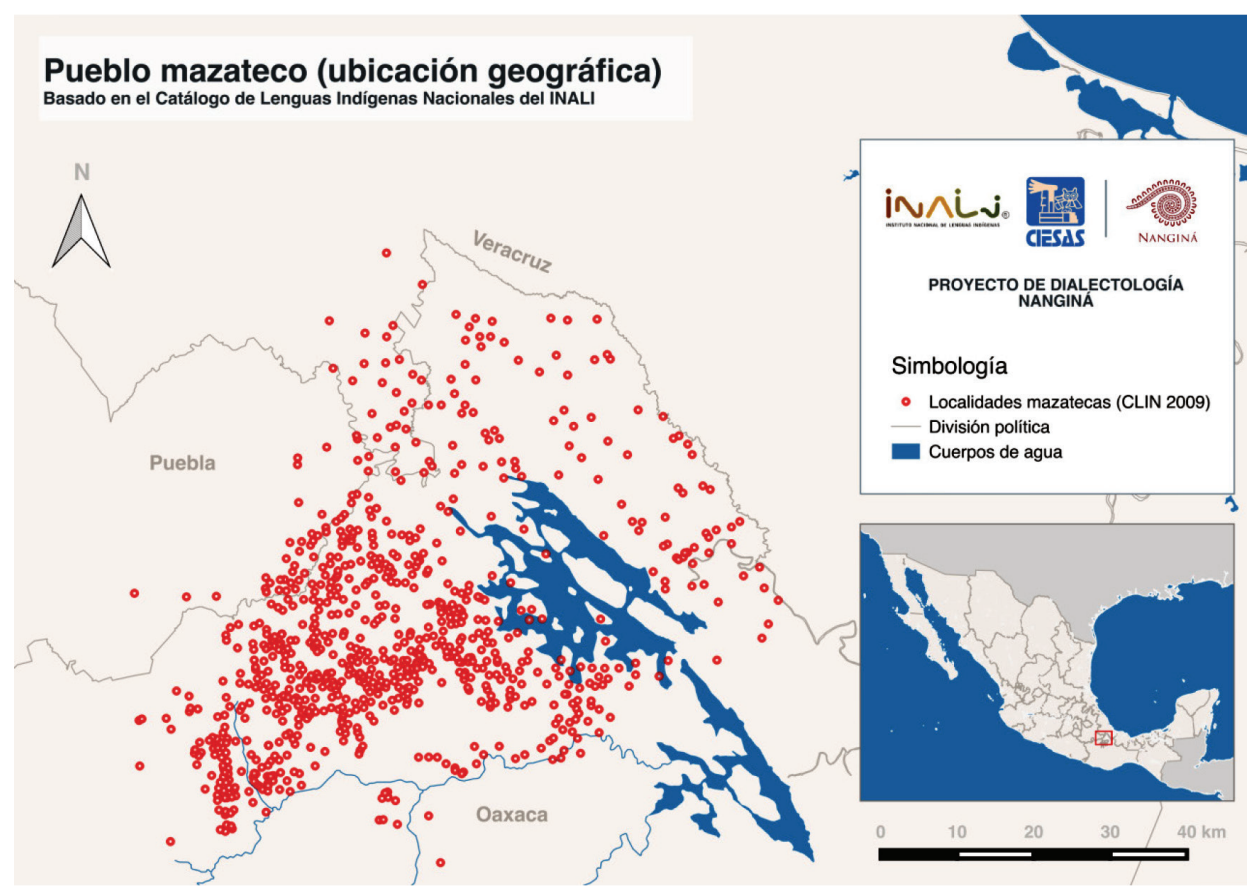

Figura 1. Pueblo mazateco ubicación geográfica.

(Mapa elaborado por Diego Mendoza).

Con base en el Proyecto Nanginá se presenta una nueva propuesta dialectal de 10 lenguas mazatecas, o zonas de inteligibilidad, y 20 variantes (ver Chávez Peón; Wagner; Filio \& García 2021). Los principales criterios de dicha propuesta son de carácter lingüístico, en relación con las diferencias y similitudes de los sonidos entre variantes, pero también tomamos en cuenta aspectos históricos y morfológicos, la división política de los municipios y la identidad comunitaria de sus hablantes. La Figura 2 muestra el mapa con esta propuesta dialectal, seguida del Cuadro 1 que recopila esta información. 


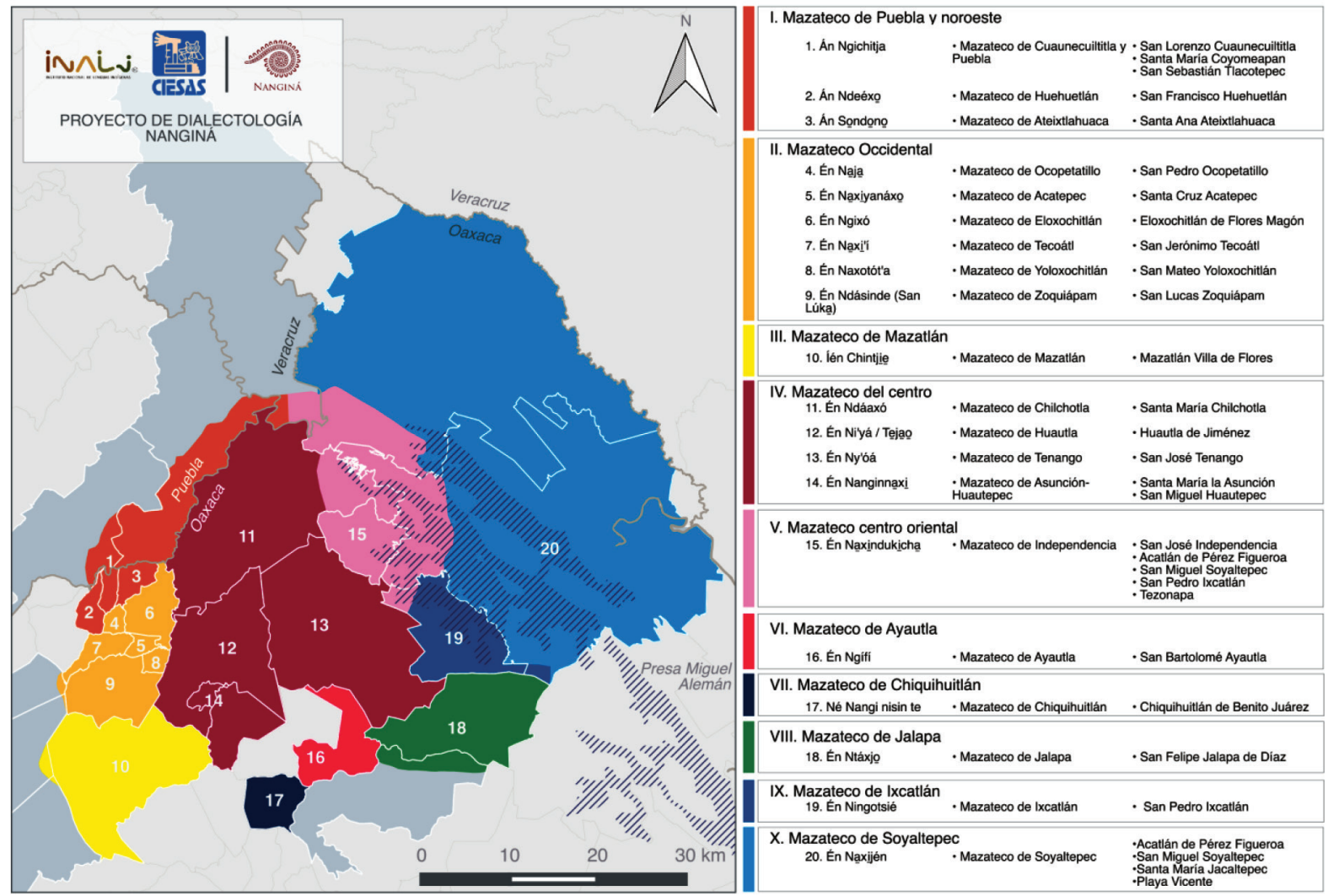

Figura 2. Propuesta dialectal de lenguas (zonas inteligibles) y variantes mazatecas. (Mapa elaborado por Diego Mendoza). 
Cuadro 1. Lista de lenguas y variantes mazatecas

\begin{tabular}{ll}
\hline LeNGUAS MAZATECAS & VARIANTES \\
\hline I. Mazateco de Puebla y del noroeste & 1. Mazateco de Cuaunecuiltitla y Puebla \\
& 2. Mazateco de Huehuetlán \\
& 3. Mazateco de Ateixtlahuaca \\
II. Mazateco occidental & 5. Mazateco de Ocopetatillo \\
& 6. Mazateco de Eloxochitlán \\
& 7. Mazateco de Tecóatl \\
& 8. Mazateco de Yoloxochitlán \\
& 9. Mazateco de Zoquiapam \\
III. Mazateco de Mazatlán & 10. Mazateco de Mazatlán \\
IV. Mazateco del Centro & 11. Mazateco de Chilchotla \\
& 12. Mazateco de Huautla \\
13. Mazateco de Tenango \\
V. Mazateco Centro Oriental & 14. Mazateco de Asunción-Huautepec \\
VI. Mazateco de Ayautla & 16. Mazateco de Ayautla \\
VII. Mazateco de Chiquihuitlán & 17. Mazateco de Chiquihuitlán \\
VIII. Mazateco de Jalapa & 18. Mazateco de Jalapa \\
IX. Mazateco de Ixcatlán & 19. Mazateco de Ixcatlán \\
X. Mazateco Oriental & 20. Mazateco de Acatlán-Soyaltepec \\
\hline
\end{tabular}

Los datos de este trabajo se presentan con base en esta propuesta dialectal, así como las descripciones y generalizaciones que se emitan. Las principales fuentes para los ejemplos presentados son el Pro-

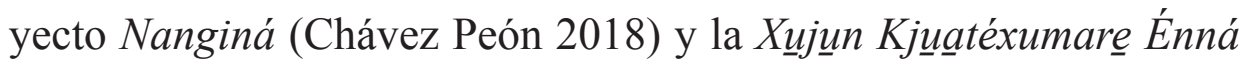
(Norma de escritura del mazateco). Estos resultados responden a 
muchos años de investigación del mazateco, no solo de los trabajos citados, sino también de valiosas investigaciones previas a nivel dialectal, incluyendo Gudchinsky (1958), Kirk (1966, 1970), Léonard, dell'Aquila, \& Gaillard-Corvaglia (2012) y Léonard, Patriarca, Sharma \& Chakraborti (2017), entre otros.

Con respecto a las descripciones lingüísticas del mazateco como agrupación, muchos trabajos se han enfocado en la compleja fonología de estas lenguas (ver Vielma 2017 para un panorama de estos estudios). Sin que nuestro objetivo sea presentar una descripción segmental exhaustiva, la presentación fonotáctica de las lenguas mazatecas requiere detallar el inventario vocálico y consonántico panmazateco. Algunos de los estudios previos a esta compilación incluyen las variantes de Huautla de Jiménez (Pike \& Pike 1947; García 2013); San Miguel Soyaltepec (Pike 1956; Beal 2011); Chiquihuitlán de Benito Juárez (Jamieson 1977; González 2021); San Jerónimo Tecóatl (Bull 1984; Agee, 1993); Jalapa de Díaz (Silverman, Blankenship, Kirk \& Ladefoged 1995); Santa Clara, Huautla de Jiménez (Herrera 2003); Mazatlán Villa de Flores (Filio 2011; 2014); San Lorenzo Cuaunecuiltitla (Carrera 2012; 2014); San Pedro Ixcatlán (Laminan 2013; Wagner 2018) y San Bartolomé Ayautla (Nakamoto 2020), así como la Norma de escritura del mazateco. Con base en estos estudios y las propuestas de los autores de este trabajo retomaremos el inventario general de las lenguas mazatecas y los análisis relevantes para explicar la estructura silábica.

El Cuadro 2 muestra los timbres vocálicos y el contraste oro-nasal panmazateco. 
Cuadro 2. Inventario de vocales del panmazateco

\begin{tabular}{|c|c|}
\hline orales & $\begin{array}{l}\text { i, e, a, o (todas); } \\
\text { u (Mazatlán, Ayautla, Independencia...) } \\
\text { æ (Jalapa), ع (Chiquihuitlán y Soyaltepec) } \\
\text { i/u (Puebla y noroeste) }\end{array}$ \\
\hline nasales & $\tilde{1}, \tilde{\mathrm{e}}, \tilde{\mathrm{a}}, \tilde{\mathrm{o}} ; \tilde{\mathrm{u}} ; \tilde{\varepsilon} / \tilde{\mathfrak{z}} ; \tilde{\mathrm{t}} / \tilde{\mathrm{u}}$ \\
\hline
\end{tabular}

A partir de la propuesta del protomazateco de 4 vocales: /*i, æ, a, u/ de Wagner (2021; cf. Gudschinsky 1956 y Kirk 1966), actualmente todos los sistemas mazatecos cuentan con al menos las 4 vocales / i, e, a, o /, siendo este el inventario de varios municipios como Huautla o Ixcatlán. Además de estas 4 vocales, varios sistemas cuentan con el fonema /u/, la mayoría fuera de la zona alta, como Mazatlán, Ayautla e Independencia, entre otros, que forman un triángulo vocálico de 5 timbres. Para algunos sistemas la /u/ es mucho más prominente que la vocal /o/, al grado de que la vocal media tenga un status fonémico defectivo como en Soyaltepec. ${ }^{1}$ Además de estos sistemas de 5 vocales, el máximo es de 6 timbres, como en los sistemas de Chiquihuitlán, Jalapa y Soyaltepec, donde se mantiene una vocal semi-abierta anterior $(\varepsilon / æ)$. Los municipios de Puebla y del noroeste han innovado con el desarrollo de una vocal central: ya sea la i herida (para la

${ }^{1}$ Beal (2011: 102-104) incluye a la vocal media posterior, /o/, en la descripción del inventario de Soyaltepec y presenta pares mínimos con la /u/; sin embargo, nos parece un fonema muy marcado en comunidades tradicionales del municipio, no así en la cabecera, Temascal, que cuenta con migración de otras variantes y una diversa realidad sociolingüística. 
mayoría) o la u herida en Ateixtlahuaca, lo anterior como consecuencia de un movimiento vocálico en cadena en todo el sistema (Wagner, Manzano \& Chávez Peón 2017). En cada lengua, los timbres orales se corresponden simétricamente con vocales nasales.

En cuanto a la complejidad del núcleo silábico, además del contraste oro-nasal, existen también tipos de fonación contrastivos, como la voz laringizada en varias de las lenguas mazatecas o la voz murmurada en el mazateco de Jalapa, contrastes que discutimos más adelante, así como la existencia de diptongos y triptongos según la variante. La existencia de estas secuencias vocálicas, incluyendo su desarrollo histórico, distribución y variación diatópica, es en sí mismo un tema de investigación a futuro que queda fuera del objetivo de este trabajo.

Las lenguas mazatecas son lenguas tonales, los sistemas cuentan con 3 o 4 tonos de nivel básicos más secuencias de contorno a partir de estos tonos. Las lenguas de la zona alta suelen presentar 4 tonos de nivel, como Zoquiapam, frente a 3 en las zonas media y baja, como Mazatlán o Jalapa, ilustrado en los siguientes ejemplos (Norma de escritura del mazateco: 62; en Chávez Peón \& Hernández 2021 puede consultarse un análisis fonético de estos tonos):

(1) a. 4 tonos de nivel (mazateco de Zoquiapam)

$\begin{array}{lll}\text { Alto } & / \mathrm{he}^{4} / & \text { 'culpa' } \\ \text { Semi-alto } & / \mathrm{he}^{3} / & \text { 'él' } \\ \text { Medio } & / \mathrm{he}^{2} / & \text { 'grande' } \\ \text { Bajo } & / \mathrm{he}^{1} / & \text { 'jilote' }\end{array}$


b. 3 tonos de nivel (mazateco de Mazatlán)
Alto $/ \mathrm{Ja}^{3} /$ 'trabajo'
Medio / $\mathrm{Ja}^{2} /$ 'tigrillo'
Bajo / $/ \mathrm{Ja}^{1} /$ 'sucio, borroso'

Respecto a las consonantes, las lenguas mazatecas cuentan con segmentos simples, complejos (un rasgo secundario) y sobre-complejos (dos rasgos secundarios). Los complejos incluyen sonidos prenasalizados, aspirados y glotalizados, mientras que los sobre-complejos pueden agrupar prenasalizadas (pos)aspiradas, así como prenasalizadas (pre)glotalizadas. Estos sonidos se compilan en el Cuadro 3 (los sonidos entre paréntesis ocurren básicamente en préstamos).

La alternancia de la fricativa posalveolar sorda $/ \mathrm{f} / \mathrm{y}$ su contraparte retrofleja /s/ divide a la zona alta, con el fonema retroflejo, del resto de las variantes (zona media y baja). El resto de los fonemas africados retroflejos pertenecen solo a la zona alta (marcados en rojo). Otros fonemas predominantemente de esta zona o de algunas variantes de esta zona son la fricativa posaspirada / $\mathrm{s}^{\mathrm{h}} /$, las resonantes preaspiradas / ${ }_{\beta} /{ }^{h} \mathrm{w}, \mathrm{h}_{\mathrm{j}}$ / (la labial solo en Huautla-Tenango), así como la lateral preglotalilzada / '1 / (particularmente en Zoquiapam, por ejemplo / 'li / 'fuego').

Por el contrario, las oclusivas y africadas posglotalizadas se concentran en la zona baja (marcadas en azul), particularmente en el continuum territorial de San José Independencia, Ixcatlán y AcatlánSoyaltepec. 
Cuadro 3. Inventario de consonantes (compartidas) del panmazateco

\begin{tabular}{|c|c|c|c|c|c|c|}
\hline & Oclusivas & AfricaDAS & FRICATIVAS & Nasales & $\begin{array}{c}\text { LíQui- } \\
\text { DAS }\end{array}$ & $\begin{array}{l}\text { APROXI- } \\
\text { MANTES }\end{array}$ \\
\hline simples & p) $\mathrm{t} \mathrm{k}$ ? & $\widehat{\mathrm{t} f}$ & $\phi \mathrm{s} \int / \mathrm{s} h$ & $\mathrm{~m} \mathrm{n} \mathrm{n}$ & $1 \mathrm{f}(\mathrm{r})$ & $\beta / \mathrm{w} \mathrm{j}$ \\
\hline
\end{tabular}

Complejas

Prenasalizadas

$\left({ }^{m b}\right){ }^{n} d{ }^{n g} \quad{ }^{n} \widehat{d z} \quad{ }^{n} \widehat{d z} \quad{ }^{n} \widehat{d z}$

Aspiradas

th $\mathrm{k}^{\mathrm{h}} \mathrm{Ts}^{\mathrm{h}} \mathrm{tf}^{\mathrm{h}} \mathrm{Ts}^{\mathrm{h}}$

Glotalizadas

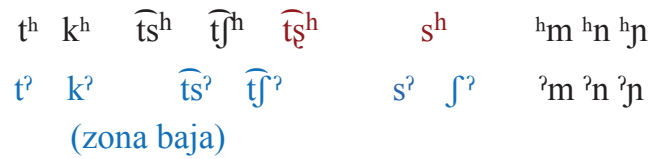

${ }^{\mathrm{h}} \beta{ }^{\mathrm{h}} \mathrm{W}_{\mathrm{W}} \mathrm{h}_{\mathrm{j}}$

${ }^{2} \beta /{ }^{\prime} \mathrm{W}^{\mathrm{j}} \mathrm{j}$

(zona baja)

Sobre-complejas

Prenasalizadas

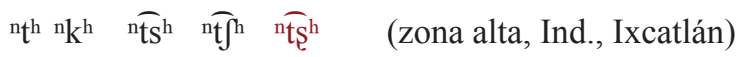

(pos)aspiradas

Prenasalizadas $\quad{ }^{\text {?mb }}{ }^{2 n} \mathrm{~d}{ }^{2 n} \mathrm{~g} \quad{ }^{2 n} \widehat{\mathrm{dz}}{ }^{{ }^{2 n} \widehat{\mathrm{d} z}}{ }^{2 n} \widehat{\mathrm{d} z} \quad$ (zona alta e Independencia)

(pre)glotalizadas

Los fonemas sobre-complejos son más restringidos que el resto, se incluyen en el Cuadro 3 porque aparecen en varias zonas, pero no son fonemas presentes en todas las variantes. Las prenasalizadas (pos) aspiradas tienen una extensión mayor que las prenasalizadas (pre)glotalizadas; estas consonantes también se presentan en la zona alta, además de Independencia e Ixcatlán, aunque en este último caso, están marcadas generacionalmente y en convivencia con nasales silábicas (ver Wagner 2018: 112, 263-264). Las prenasalizadas (pre)glotalizadas se registran en la zona alta y fuera de ella, únicamente en Independencia. 
Además de los fonemas del Cuadro 3, es necesario mencionar varias particularidades de distintas lenguas y variantes mazatecas. Por ejemplo, la zona de Puebla y del noroeste resulta la más innovadora a nivel segmental, con cambios vocálicos notorios, como se mencionó arriba, y una particular innovación en las oclusivas y africadas, con un cambio en curso que incluye, además de consonantes sordas, aspiradas y prenasalizadas, también incluye sonoras: / d, g, dz, $\widehat{\mathrm{d} z}$ / (ver Wagner 2017; Ly Van Tu 2015).

Las lenguas mazatecas suelen mostrar una variación complementaria entre la lateral y la vibrante. La zona del centro usa la lateral preferentemente, mientras Mazatlán, Independencia o Chiquihuitlán cuentan con la vibrante como fonema.

Para el mazateco de Ixcatlán, el detallado estudio fonológico de Wagner (2018), incluye, además de consonantes aspiradas, una serie de segmentos glotalizados, palatalizados y labializados, que la convierten al día de hoy en la lengua mazateca con el mayor inventario descrito, con 70 fonemas (Wagner 2018: 50). El mazateco de Chiquihuitlán cuenta también con una serie de consonantes palatalizadas; mientras que otras descripciones que reportan sonidos labializados incluyen a Ayautla (Nakamoto 2020).

Los segmentos labiales cuentan con un estatus especial dentro de las lenguas mazatecas (y en general las lenguas otomangues). Dada la inexistencia de oclusivas labiales en el protomazateco, los segmentos / $\mathrm{p} / \mathrm{y} / \mathrm{mb} /$ ocurren básicamente en préstamos del español.

La aproximante labial / $\beta_{\mathrm{r}} / \mathrm{o} / \mathrm{w} /$, con variación en el redondeamiento y la posterioridad según la descripción, proviene del 
protofonema ${ }^{*}$ (ver discusión en García 2013: 52-54). Finalmente, en algunas lenguas, como Ixcatlán (Wagner 2018: 77) y Huautla (García 2013: 54) se reporta también la fricativa bilabial sorda / $\phi$ / ( $/ \mathrm{h} \beta /$ en Tenango; fonemas que también derivan de la ${ }^{*} \mathrm{w}$ ). La variación y alofonía de los segmentos labiales en las lenguas mazatecas se ha documentado para distintas variantes. Tanto Léonard \& Fulcrand (2016) como Wagner (2018) documentan el betacismo de *w a / $\beta / \mathrm{b} /$.

Nakamoto (2020) reporta para Ayautla consonantes lamino alveo-palatales: /t $6,6,{ }^{n_{t}}\left[{ }^{n} \mathrm{~d} / \mathrm{t}\right] /$, no descritas previamente en otros sistemas y con una distribución restringida según el autor. Algunas de estas características no se discuten a profundidad en este trabajo y se remite al lector a las referencias arriba mencionadas.

Con base en el macro-sistema segmental presentado -inédito en cuanto al análisis global de estas lenguas-, nos alineamos a trabajos que proponen un sistema consonántico complejo y una estructura silábica relativamente simple (Silverman, Blankenship, Kirk \& Ladefoged 1995; Golston \& Kehrein 1998; García 2013; Carrera 2014; Filio 2014; Wagner 2018), frente a otros trabajos que, por el contrario, proponen un sistema consonántico relativamente simple pero una estructura silábica compleja (Pike \& Pike 1947; Kirk 1966; Jamieson 1977; Lamiman 2013; Beal 2011; Nakamoto 2020). Este análisis radica sobre todo en la posibilidad de describir y predecir articulaciones secundarias con base en la teoría de rasgos distintivos, como se detallará más adelante para cada clase natural.

Todas las descripciones de las lenguas mazatecas coinciden en la inexistencia de codas, es decir, la prohibición de consonantes a final 
de sílaba; mientras que sobre los inicios o ataques silábicos hay mayor disparidad de análisis. Los puntos de mayor diferencia son el análisis de los gestos nasales y los rasgos laríngeos en adyacencia con consonantes. La distribución de estos rasgos constituye un punto esencial para determinar la fonotáctica de estas lenguas, pues a partir de su estatus fonológico se deducen muchas de las secuencias permitidas y su constitución silábica. Como tal, requieren una descripción inicial y su análisis se retomará en distintos puntos del artículo. Sobre los gestos nasales, como muestra el inventario panmazateco, asumimos la existencia de consonantes prenasalizadas, en línea la mayoría de las descripciones. Algunos trabajos, sin embargo, describen los gestos nasales + oclusión como una secuencia CC (Pike \& Pike 1947; Beal 2011).

Todos los trabajos reportan la secuencia de fricativa más obstruyente en diferentes lenguas mazatecas, descripción que se extiende en los trabajos de Filio (2014) y García (2013) a la existencia de secuencias de la fricativa glotal más consonantes prenasalizadas, como veremos más adelante. Los trabajos que indagan en mayor medida sobre la fonotáctica mazateca o estructura silábica de variantes en particular son las tesis sobre Soyaltepec (Beal 2011), Huautla (García 2013), Mazatlán (Filio 2014), Ixcatlán (Wagner 2018) y Ayautla (Nakamoto 2020). Varios puntos de estos trabajos se retoman a lo largo del presente artículo. Referencias anteriores que aludan a la estructura silábica o reporten temas de fonotáctica incluyen a Pike \& Pike (1947), Kirk (1966), Jamieson 1977, y Gregorio (1993).

Como punto de partida en relación a la aspiración y glotalización, a priori presentamos de manera descriptiva sonidos "pre" y "pos" 
aspirados, así como "pre" y "pos" laringizados del mazateco de Huautla (García 2013: 66), antes de definir el estatus fonológico de estos rasgos.
a. [ ${ }^{\mathrm{ta}^{1}}$ ] 'voz'
b. [ $\left.\mathrm{t}^{\mathrm{h}^{1}}\right]$ 'ligero'
c. [ $\left.{ }^{\text {jja }}{ }^{1}\right]$ 'arcoíris'
d. $\left[\mathrm{j}^{\mathrm{P}} \mathrm{a}^{3}\right]$ 'lo trae'

Sobre este tipo de gestos laríngeos, la primera propuesta teórica sobre el debate de la estructura silábica en mazateco fue la investigación de Pike \& Pike (1947), realizada también para la variante de Huautla de Jiménez (zona centro). Estos autores analizan la sílaba a partir de la división de sus elementos en constituyentes inmediatos (como una analogía sintáctica). Así, los inicios complejos son capas que se estructuran por un miembro principal con elementos subordinados, incluyendo los gestos de nasalidad, aspiración y glotalización, que forman grupos de dos o tres consonantes en un inicio, por ejemplo: /ntho/ 'aire' o /hngo/ 'uno'. Para la palabra [nts'wajl] 'nuestros (excl.) estómagos', los autores proponen el esquema en la Figura 3.

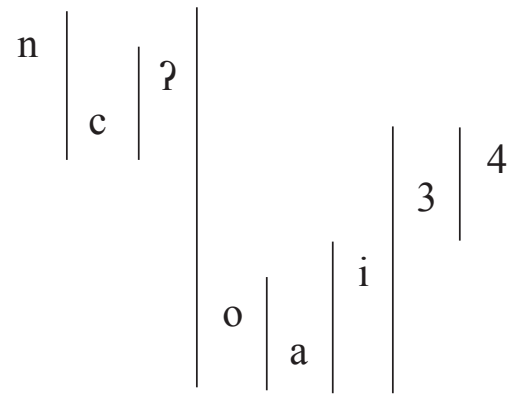

Figura 3. Esquema de constituyentes inmediatos en mazateco de Pike \& Pike (1947: 91) 
La Figura 3 muestra una división de constituyentes en núcleo y márgenes, es decir vocales versus consonantes, más los tonos. En este ejemplo, la africada alveolar sorda /c/ (representada con el Alfabeto Fonético Americano; /ts/ en el Alfabeto Fonético Internacional) constituye el elemento principal del inicio silábico mientras que la glotal y la nasal son elementos subordinados.

Esta propuesta sobresaliente, claramente apuntaba, por un lado, a análisis posteriores de gradas consonánticas, vocálicas y tonales desarrolladas en trabajos como Goldsmith (1976) y Clements \& Keyser (1983); por el otro, esbozaba también las relaciones jerárquicas entre diferentes gestos articulatorios, postulados como secuencias de segmentos en Pike \& Pike (1947), pero reanalizados como rasgos secundarios en trabajos posteriores (por ejemplo, en la geometría de rasgos de Clements 1985).

Casi cinco décadas después este trabajo fue retomado por Steriade (1994), quien propuso los inicios complejos (rasgos laringizados) como grupos de fonemas subyacentes que se fusionan para formar un solo segmento fonético (un grupo de tres segmentos ligados C?V y $\mathrm{ChV}$ y un grupo de dos segmentos con un inicio simple + un segmento complejo $\mathrm{C}^{\mathrm{P}} \mathrm{V}$ y $\mathrm{C}^{\mathrm{h}} \mathrm{V}$ ). Con cierta similitud de análisis, Nakamoto (2020: 105-106) reporta en la fonotáctica del mazateco de Ayautla secuencias triconsonánticas conformadas de una sibilante más plosiva más consonante glotal, así como una secuencia cuadriconsonántica: sibilante-plosiva-glotal-yod.

Sobre esta discusión, los trabajos de Golston \& Kehrein (1998) y Silverman, Blankenship, Kirk \& Ladefoged (1995) proponen que esta 
complejidad puede repartirse entre los inicios y los núcleos silábicos. Los casos que presentan post-aspiración y post-glotalización los consideran en realidad como voz murmurada o voz laringizada, mientras que la pre-aspiración y pre-glotalización como rasgos laríngeos de la consonante inicial.

Otro análisis para este fenómeno, lo presentan Klint \& Filio (2018) para el mazateco de Mazatlán, quienes proponen que la aspiración y glotalización son rasgos del morfema. Este análisis, si bien novedoso y utilizado para otras lenguas otomangues (por ejemplo, para el mixteco, Marlett 1992: 425), no responde cabalmente a todos los ejemplos de las lenguas mazatecas, por la distribución y morfología de algunos casos. No obstante, es un parámetro que merece mayor investigación a futuro.

García, Chávez Peón \& Polian (2012), así como García (2013), retoman este importante debate teórico y descriptivo sobre el estatus de los rasgos laríngeos de las lenguas mazatecas. Con datos de la comunidad de Río Santiago, Huautla de Jiménez. Esquemáticamente plantean la sílaba del mazateco de Huautla de la siguiente manera.

(3) Sílaba del mazateco de Huautla:

$\{\mathbf{h} / \mathbf{p}\}-\mathbf{n}-\mathbf{C}-\{\mathbf{h} / \mathbf{p}\}-\mathrm{V}$

rasgo lar. prenas. cons. rasgo lar. vocal (+/-nasal, mono/di/tri-ptongo)

Se postula que la sílaba del mazateco se compone, obligatoria y mínimamente, de una consonante y una vocal, más la posible presencia de rasgos laríngeos "pre” o "pos" consonánticos, en los que 
centran una discusión y detallada descripción de sus datos. Los autores proponen que cuando existe una aspiración antes de una consonante se trata de un inicio complejo (secuencia consonántica) y cuando la aspiración va después de una consonante se manifiesta una consonante aspirada (segmento complejo). En cuanto al gesto laríngeo glotalizado, cuando se antepone a una consonante (solo resonantes), se trata de una consonante glotalizada (segmento complejo); y cuando la glotalización se presenta después de la consonante, el rasgo laríngeo pertenece al núcleo silábico, como un rasgo vocálico de voz laringizada. En (4) retomamos los ejemplos presentados (2) bajo este análisis, seguidos de (5) y (6), que sintetizan esta propuesta (García, Chávez Peón \& Polian 2012; García 2013).
a. /htal/ 'voz'
b. $/ \mathrm{t}^{\mathrm{h}} \mathrm{a}^{1 /}$ 'ligero'
c. $/ \mathrm{ja}^{1 /}$ 'arcoíris'
d. $/ \mathrm{ja}_{\mathrm{a}}^{3} /$ 'lo trae'

(5) a. hC "pre-aspirado" 2 inicio complejo = secuencia consonántica. b. Ch "pos-aspirada" segmento complejo = consonante aspirada.

(6) a. ${ }^{2} \mathrm{C}$ "pre-glotalizada" segmento complejo $=$ consonante laringizada. b. CV "pos-glotalizada" voz laringizada = rasgo vocálico.

2 Las comillas para los términos "pre" y "pos" se utilizan con la finalidad de hacer un acercamiento preliminar descriptivo de los datos, sin análisis ni conclusiones teóricas. 
Lo anterior sigue el principio de ligamiento de Kingston (1985; ver también Howe \& Pulleyblank 2001; Kehrein \& Golston 2004), que estipula que las oclusivas presentan rasgos secundarios a la derecha ("pos"), es decir, en su soltura; mientras que las resonantes, preferentemente realizan rasgos secundarios a la izquierda ("pre") como se detalla más adelante.

Para este trabajo, retomamos este análisis de la variante de Huautla a la luz de nuevos datos y estudios de todas las lenguas mazatecas; sin embargo, realizaremos varias precisiones y diferencias, sobre todo en comparación con la zona baja, donde sí existen fonemas obstruyentes posglotalizados. Además, acotaremos una importante distinción entre obstruyentes y resonantes como clases naturales. Con base en este análisis veremos que en todas las lenguas mazatecas se cumple la restricción fonotáctica principal ya anticipada de permitir hasta dos consonantes en inicio y que en palabras nativas esta secuencia se restringe a fricativa + plosiva.

\section{INICIOS SIMPLES}

Como hemos mencionado ya, la plantilla silábica básica (no única) de las lenguas mazatecas es CV. Como tal, todos los fonemas pueden aparecer a principio de palabra en la formación de sílabas iniciales CV. Así, una de las principales características de la sílaba mazateca es que el inicio consonántico es obligatorio. Este principio se ha confirmado en varios trabajos, incluyendo García (2013), Filio (2014), 
Wagner (2018) y Nakamoto (2020). Esto incluye a la oclusiva glotal que ocurre a inicio de palabra (aunque en esta posición no se escriba a nivel ortográfico). Dependiendo de la variante, esta oclusiva se mantiene también en palabras compuestas, como se ilustra en (7), para el mazateco de Ayautla (Nakamoto 2020: 84).

$$
\begin{aligned}
& \begin{array}{ll}
\text { a. } i x i ̈ & \text { b. nda'íxï }
\end{array} \\
& \mathrm{Pi}^{2}{ }^{2} \mathrm{i}^{23} \quad{ }^{\mathrm{n}} \mathrm{ta}^{2} \mathrm{Pi}^{4}{ }^{\prime} \mathrm{ci}^{231} \\
& { }^{n} \mathrm{ta}^{3(4)}+\mathrm{Pi}^{2}{ }^{2} \mathrm{i}^{23=1} \\
& \text { agua }+ \text { dulce }=\text { ABS } \\
& \text { 'dulce' 'refresco' }
\end{aligned}
$$

Para reforzar el argumento de la glotal a principio de palabra y de la obligatoriedad del inicio silábico, García (2013: 62-65) propone también analizar a la glotal intervocálica como consonante, ocupando la posición de inicio, es decir, postula la inexistencia de vocales rearticuladas en el mazateco de Huautla (contrario, por ejemplo, al análisis de esta glotal como vocal rearticulada fonológica en lenguas zapotecas, como se expone en Chávez Peón (2010: 212-214) y referencias ahí citadas; cf. González (2021) y su análisis para este fenómeno en el mazateco de Chiquihuitlán).

En el presente trabajo asumimos también la obligatoriedad del inicio a nivel silábico, la epéntesis de una oclusiva glotal fonética en palabras que inician con vocal y la existencia de la glotal como consonante intervocálica (a diferencia de analizarla como vocal rearticulada). 
Una vez definido este principio de inicio obligatorio para las lenguas mazatecas, mostraremos la diversidad de inicios en relación al modo de articulación de diferentes consonantes. Asimismo, haremos uso de la teoría de rasgos distintivos (Jakobson, Fant \& Halle 1952; Chomsky y Halle 1968; Clements 1985; Clements \& Hume 1995; entre otros) para ilustrar las diferentes clases naturales que ocupan la posición de inicio. Estas características distintivas, a su vez, se han organizado jerárquicamente de manera arbórea, bajo la propuesta de la "Geometría de los rasgos distintivos" (Clements 1985; Hall 2007, entre otros).

Para ilustrar todas las clases naturales del mazateco, nos centraremos en las consonantes coronales, con las que podemos mostrar todos los modos de articulación y combinación de rasgos distintivos.

\subsection{Oclusivas y africadas (plosivas)}

La primera clase natural que presentamos son las oclusivas, segmentos que se caracterizan por presentar un cierre total en la cavidad oral, con lo que se impide el paso del aire momentáneamente, para después liberarlo a modo de soltura de los articuladores. Este modo de articulación, se define con base en los rasgos distintivos: [+consonántico, -resonante, -continuo]. Dentro de nuestra perspectiva, las africadas se agrupan también dentro de las oclusivas como clase natural (en línea con Clements 1999, quien agrega el rasgo [+estridente] para dichos segmentos). Nos referiremos, como se mencionó arriba, de manera conjunta a oclusivas y africadas como plosivas (aunque en diferentes 
apartados se utiliza el término oclusiva de manera general por ser más común). A la par de la composición interna básica de estos segmentos, las lenguas mazatecas cuentan también con rasgos secundarios que agregan diferentes distinciones para crear un mayor número de contrastes, incluyendo [+nasal] para las prenasalizadas y rasgos laríngeos de aspiración [+glotis extendida] y glotalización [+glotis constreñida], dependiendo de la variante, como veremos más adelante.

A continuación, se muestra la clase natural de las plosivas simples encontradas en las lenguas mazatecas, seguidos de ejemplos con la / t/.

(8) Plosivas (oclusivas y africadas): clase natural [+consonántico, -resonante, -continua]

Lista de fonemas plosivos simples: / (p), t, k, ?, $\overparen{\mathrm{ts}}, \widetilde{\mathrm{t} f}, \widehat{\mathrm{ts}} /$

\section{Cuadro 4. Palabras con $/ \mathrm{t} /$}

\begin{tabular}{|c|c|}
\hline EJEMPLO & Zona \\
\hline$/$ to $^{1 /}$ 'voz' & Coyomeapan, Tlacotepec, Huehuetlán, Cuaunecuiltitla, Ateixtlahuaca \\
\hline$/ \mathrm{ti}^{1} /$ 'pescado' & $\begin{array}{l}\text { Ocopetatillo, Eloxochitlán, Tecóatl, Acatepec, Yoloxochitlán, Toxpa- } \\
\text { lan, Los Cués, Zoquiapam, Mazatlán, Tecomavaca; Independencia, } \\
\text { Tezonapa, Chiquihuitlán, Ixcatlán, Soyaltepec, Acatlán }\end{array}$ \\
\hline$/ \mathrm{ti}^{2} /$ 'niño' & Chilchotla, Huautla, Asunción, Huautepec, Tenango, Jalapa, Teutila \\
\hline$/ \mathrm{te}^{2} /$ 'amplio' & Ayautla \\
\hline
\end{tabular}

En contraste con las oclusivas simples, todas las variantes mazatecas presentan también oclusivas prenasalizadas, contrastando con las simples precisamente por el rasgo [+nasal] que como consecuencia 
sonoriza a la oclusiva (cf. Carrera 2014 y su propuesta de nasales posoclusivizadas). A continuación, mostramos los segmentos de esta clase y algunos ejemplos. (Fonológicamente, algunos trabajos representan estos sonidos como / $\mathrm{n}_{\mathrm{t}}$, Pike \& Pike 1947; Nakamoto 2020.)

(9) Plosivas prenasalizadas [+consonántico, -resonante, -continua, +nasal]

Lista de fonemas oclusivos prenasalizados (incluyendo africadas): /mb, ${ }^{\mathrm{n}} \mathrm{d},{ }^{\mathrm{n}} \mathrm{g},{ }^{\mathrm{n}} \widehat{\mathrm{dz}}{ }^{\mathrm{n}} \widehat{\mathrm{dz}}{ }^{\mathrm{n}} \widehat{\mathrm{dz}} /$

Cuadro 5. Palabras con $/ \mathbf{n}^{\mathrm{d} /}$

\begin{tabular}{|c|c|}
\hline EJEMPLO: 'BUENO' & ZoNa \\
\hline$/ \mathrm{do}^{2} \mathrm{ho}^{3} /$ & $\begin{array}{l}\text { Coyomeapan, Tlacotepec, Huehuetlán, Cuaunecuiltitla, Ateixtla- } \\
\text { huaca }\end{array}$ \\
\hline$/ \mathrm{da}^{2} /$ & $\begin{array}{l}\text { Ocopetatillo, Eloxochitlán, Tecóatl, Acatepec, Yoloxochitlán, Tox- } \\
\text { palan, Los Cués, Zoquiapam, } \\
\text { Mazatlán, Tecomavaca, Chilchotla, Huautla, Asunción, Huautepec, } \\
\text { Tenango, Independencia, Tezonapa, Jalapa, Teutila, Ixcatlán }\end{array}$ \\
\hline$/ \mathrm{da}^{3} /$ & Ayautla \\
\hline$/{ }^{\mathrm{n}} \mathrm{da}^{1} \mathrm{ha}^{3} /$ & Chiquihuitlán \\
\hline$/{ }^{\mathrm{n}} \mathrm{da}^{2} \mathrm{hi}^{3} /$ 'caña' & Soyaltepec, Acatlán \\
\hline
\end{tabular}

Una vez presentados los ejemplos de oclusiva simple y prenasalizada, pasemos a la gran complejidad de los fonemas mazatecos: sus rasgos laríngeos, mencionados ya anteriormente. La aspiración se codifica con el rasgo [+glotis extendida] (gesto articulatorio que abre la glotis y permite el libre paso del aire por la laringe hacia el tracto 
vocálico), mientras la glotalización se codifica con el rasgo [+glotis constreñida] (gesto que implica una fuerte constricción en la laringe). (A nivel ortográfico, la aspiración se escribe con una $j$, mientras que para la glotalización se utiliza la apóstrofe '). A continuación, presentamos las consonantes plosivas aspiradas del panmazateco.

(10) Plosivas aspiradas [+consonántico, -resonante, -continua, +glotis extendida]

Lista de fonemas oclusivos aspirados (incluyendo africadas): $/ \mathrm{t}^{\mathrm{h}}, \mathrm{k}^{\mathrm{h}}, \widehat{\mathrm{ts}}^{\mathrm{h}}, \widehat{\mathrm{t}}^{\mathrm{h}}, \widehat{\mathrm{ts}}^{\mathrm{h}} /$

Cuadro 6. Palabras con / $\mathrm{t}^{\mathrm{h}} /$

\begin{tabular}{|c|c|}
\hline EJEMPLO & ZoNa \\
\hline$/ \mathrm{t}^{\mathrm{h}} \mathrm{1}^{4} /$ 'hay' & $\begin{array}{l}\text { Coyomeapan, Tlacotepec, Huehuetlán, Cuaunecuiltitla, Ateixtla- } \\
\text { huaca }\end{array}$ \\
\hline$/ \mathrm{t}^{\mathrm{h}^{2} /}$ 'redondo' & $\begin{array}{l}\text { Ocopetatillo, Eloxochitlán, Tecóatl, Acatepec, Yoloxochitlán, Tox- } \\
\text { palan, Los Cués, Zoquiapam, Mazatlán, Tecomavaca, Chilchotla, } \\
\text { Huautla, Asunción, Huautepec, Tenango, Independencia, Tezona- } \\
\text { pa, Ayautla, Jalapa, Teutila }\end{array}$ \\
\hline$/ \mathrm{t}^{\mathrm{h}} \mathrm{O}^{1 /}$ 'viento' & Chiquihuitlán, Ixcatlán ${ }^{3}$ \\
\hline$/ \mathrm{t}^{\mathrm{h}} \mathrm{iu}^{2 \mathrm{n}} \mathrm{ga}^{2} /$ 'ala' & Soyaltepec, Acatlán \\
\hline
\end{tabular}

${ }^{3}$ De acuerdo con Wagner (2018), la mayoría de los ítems con tono bajo en la superficie no están específicados con ningún tono en la subyacencia; sin embargo, aquí se incluye por simplicidad expositiva. 
Estos datos muestran el comportamiento tipológicamente más esperable de obstruyentes aspiradas, es decir, una realización pos-aspirada. Ahora bien, anteriormente mencionamos que en el mazateco podemos también tener consonantes "pre-aspiradas", como el ejemplo de (2) [ $\left.{ }^{h} \mathrm{ta}^{1}\right]$ 'voz'; sin embargo, ante la imposibilidad teórica de que dos segmentos contrasten como pre vs. posaspirado García, Chávez Peón \& Polian (2012) y García (2013) analizan este tipo de ejemplos como una secuencia de dos consonantes, como mencionamos arriba.

(11) a. hC "pre-aspirado" inicio complejo = secuencia consonántica

b. $\mathrm{C}^{\mathrm{h}}$ "pos-aspirada" segmento complejo = consonante aspirada

En este trabajo, implementamos este esquema del rasgo de aspiración a la descripción de las obstruyentes en todas las lenguas mazatecas. Consecuentemente, los casos de aspiración más consonante, es decir secuencias como /ht/, se presentan más adelante, en la sección de inicios complejos. Asimismo, más abajo abordamos la distribución de la aspiración dentro de las resonantes.

Una vez descrito el comportamiento del rasgo [+glotis extendida], pasemos al rasgo [+glotis constreñida], relativo a la glotalización o laringización de un segmento. A nivel lingüístico (y ortográfico), las lenguas mazatecas se han descrito con una gran cantidad de sonidos con este rasgo, incluyendo consonantes glotalizadas y vocales laringizadas. Comenzamos con una descripción diacrónica y dialectal de la variación de este rasgo en las lenguas mazatecas. Gran parte de la complejidad laríngea de estas lenguas proviene de una síncopa 
vocálica en palabras del tipo ${ }^{*} \mathrm{CV}$ ?V y, posteriormente, un debilitamiento vocálico, como se muestra en la siguiente cadena evolutiva, con distintos estadios sincrónicos según diferentes variantes.

(12) Palabra bisilábica con glotal intervocálica

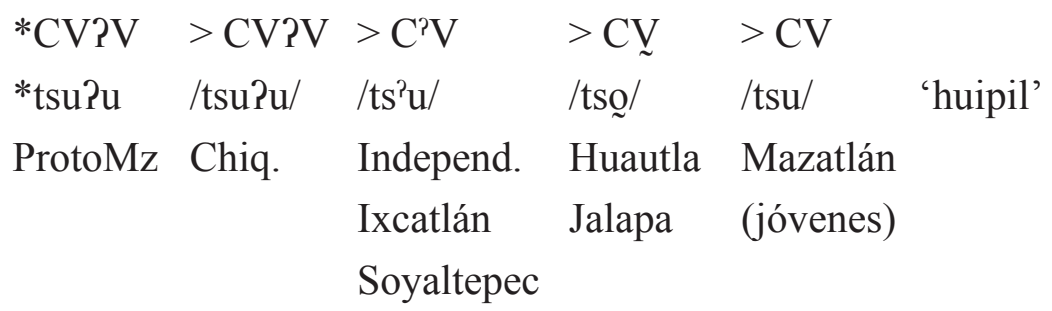

En esta cadena evolutiva incluimos la protoforma * CV?V (Kirk 1966: 232), que se mantiene en algunas palabras para ciertas variantes, como Chiquihuitlán. De ahí, en la zona baja (salvo Jalapa) la pérdida de la primera vocal (átona) provocó la adyacencia de la plosiva y la glotal, que analizamos aquí como una consonante glotalizada (en línea con Wagner 2018 para Ixcatlán). En la zona alta encontramos cierta fluctuación entre sonidos eyectivos y voz laringizada, como en Huautla, hasta llegar a la pérdida del rasgo [+g.c.] como el caso del habla juvenil de Mazatlán (Filio 2014; Klint \& Filio 2018: 4). ${ }^{4}$

4 Otra posibilidad analítica (discutida con Carlos Wagner, comunicación personal, 24 de mayo 2021) consiste en plantear dos rutas evolutivas. Una donde el debilitamiento y pérdida de la vocal pretónica provoque que la glotal se mantenga y se fusione como rasgo secundario con la consonante (caso de variantes como Independencia); la otra, que el debilitamiento de la consonante glotal deriva en voz laringizada para variantes como Jalapa 
Después de hacer un recorrido dialectal de este fenómeno con base en la propuesta de la Figura 2, para la zona I (mazateco de Puebla y del noroeste), Carrera (2014: 78) reporta voz laringizada, concretamente en el municipio de Cuaunecuiltitla. Para los demás municipios de esta zona parece haber cierta variación entre producción de eyectivas, vocales rearticuladas o consonantes glotalizadas versus voz laringizada plena, al igual que en la zona II, aunque parece predominar el tipo de voz no modal dentro de la vocal, como reporta también Martínez (2020) para Zoquiapam; la misma descripción, con predominancia de la voz laringizada, aparece en Filio (2014) para Mazatlán (zona III), y varias referencias para la zona centro (zona IV), en particular Huautla (García 2013 y referencias ahí citadas). La voz laringizada también se ha reportado con bastante evidencia en Jalapa (zona VIII; Blankenship 2002). Así, para el presente trabajo, aunque exista variación dependiendo del municipio, tomaremos de manera general la producción de voz laringizada en la zona alta y Jalapa a nivel fonológico, por ende, al ser un rasgo vocálico, queda fuera de la fonotáctica consonántica.

En contraste, para Ayautla ${ }^{5}$ (zona VI), zona de transición, así como para los municipios de Independencia (zona V), Ixcatlán (IX) y Acatlán-Soyaltepec $(\mathrm{X})$ dentro de la zona baja, se considera la existencia

(este mismo debate laríngeo existe para otras lenguas como el totonaco o variantes zapotecas). La evidencia para estos posibles análisis requiere mayor investigación a futuro.

${ }^{5}$ Nakamoto (2020) propone secuencias de plosiva más glotal (consonante + consonante), que aquí se reanalizan como oclusivas glotalizadas. 
de consonantes glotalizadas. Consonantes que se alinean a la fonotáctica de un inicio simple y se ilustran a continuación.

(13) Plosivas (pos)glotalizadas [+consonántico, -resonante, -continua, + glotis constreñida]

Lista de fonemas oclusivos (pos)glotalizados (incluyendo africadas; zona baja):

$/ \mathrm{t}^{\mathrm{P}}, \mathrm{k}^{\mathrm{P}}, \mathrm{ts}^{\mathrm{P}}, \mathrm{t} \mathrm{f}^{\mathrm{P}} /$

Cuadro 7. Palabras con $/ \mathbf{t}^{2} /$ (Ayautla y zona baja)

\begin{tabular}{ll}
\hline EJEMPLO & Zona \\
\hline$/ t^{\prime}{ }^{2} /$ 'vete' & Independencia, Tezonapa, Ayautla \\
$/ t^{2} \mathrm{e}^{2} /$ 'liso' & Ixcatlán \\
$/ \mathrm{t}^{\prime} \tilde{a}^{2} /$ ' 'vete' & Soyaltepec, Acatlán \\
\hline
\end{tabular}

El gesto de glotalización a la izquierda de las obstruyentes no existe en las lenguas mazatecas, es decir, no hay obstruyentes preglotalizadas, ni tampoco posibles secuencias de glotal + obstruyente. Más adelante se describirá la distribución del rasgo [+g.c.] en las resonantes.

Una vez analizadas las consonantes oclusivas y africadas complejas, incluyendo prenasalizadas, aspiradas y glotalizadas, pasamos ahora a las consonantes sobre-complejas, presentes sobre todo en la zona alta y, parcialmente, en la zona baja. De nueva cuenta, su descripción y análisis resulta fundamental para el tema de la fonotáctica, pues diferentes perspectivas han ofrecido diferentes resultados. En varias lenguas mazatecas podemos tener inicios silábicos con un 
gesto nasal, una oclusión y una aspiración: / $\{\mathrm{n}\}+\mathrm{t}+\{\mathrm{h}\} /$, o bien una aspiración y gesto nasal más oclusión: $/ \mathrm{h}+\{\mathrm{n}\}+\mathrm{t} /$. Desde nuestra perspectiva, y con base en la teoría de rasgos distintivos (Hall 2007), en el primer caso hablamos de un nodo raíz oclusivo que permite un rasgo secundario nasal, así como una aspiración, alineándose a la implementación de estos rasgos en segmentos complejos descritos anteriormente (prenasalizadas y posaspiradas). En este caso, por influencia de la aspiración, las oclusivas se realizan como sordas, a pesar de la prenasalización. En el caso de la aspiración más nasalización y oclusiva, hablamos de una secuencia de una fricativa glotal como $\mathrm{C} 1$ y una oclusiva prenasalizada como $\mathrm{C} 2$, que se retomarán en la siguiente sección de inicios complejos.

Con base en lo anterior, la complejidad de estos gestos articulatorios se mantiene hasta cierto grado a nivel monosegmental, continuando así con la lista de posibles inicios simples $(\mathrm{CV})$ en la fonotáctica mazateca.

(14) Plosivas prenasalizadas posaspiradas [ + cons., -resonante, -continuo, +nasal, +g.e.]

Lista de fonemas oclusivos prenasalizados posaspirados (incluyendo africadas):

$/{ }^{\mathrm{n}} \mathrm{t}^{\mathrm{h}},{ }^{\mathrm{n}} \mathrm{k}^{\mathrm{h}}, \widetilde{\mathrm{nts}}^{\mathrm{h}}, \widetilde{\mathrm{nt}^{\mathrm{h}}}, \widetilde{\mathrm{n}_{\mathrm{ts}}^{\mathrm{h}}} /$ 
Cuadro 8. Palabras con $/{ }^{n} t^{h} / 6$

\begin{tabular}{|c|c|}
\hline EJEMPLO & Zona \\
\hline$/{ }^{n} \mathrm{t}^{\mathrm{h}}{ }^{1 /}$ 'aire' & $\begin{array}{l}\text { Mazatlán, Tecomavaca, Independencia, Tezonapa, Ayautla, } \\
\text { Jalapa, Teutila }\end{array}$ \\
\hline$/{ }^{n} \mathrm{t}^{\mathrm{a}_{\mathrm{ao}}}{ }^{1 /}$ 'aire' & Chilchotla, Huautla, Asunción, Huautepec, Tenango \\
\hline$/ \mathrm{n}^{\mathrm{h}} \mathrm{e}^{2} /$ 'crepúsculo' & Ixcatlán \\
\hline
\end{tabular}

El último caso de consonante sobre-compleja se trata de una oclusiva preglotalizada prenasalizada, es decir, un gesto glotal más nasal más oclusión. De nueva cuenta, dentro de una configuración geométrica de rasgos distintivos, estas características pueden asociarse a un único nodo raíz segmental, es decir, una oclusiva o africada con dos rasgos secundarios: nasal y glotal.

(15) Plosivas preglotalizadas prenasalizadas [+cons., -resonante, -continuo, + g.c., + nasal]

Lista de fonemas oclusivos preglotalizados prenasalizados (incluyendo africadas):

$/{ }^{\text {m }} \mathrm{b},{ }^{{ }^{n} \mathrm{~d}},{ }^{2{ }^{n}} \mathrm{~g},{ }^{{ }^{n} \mathrm{dz}},{ }^{{ }_{\mathrm{n}}} \widehat{\mathrm{d}} /{ }^{7}$

${ }^{6}$ En Ixcatlán, hay una diferencia entre estos casos de una consonante oclusiva prenasalizada aspirada versus otros cuya evolución corresponde a dos sílabas, con una nasal silábica seguida de una consonante aspirada. Esto es resultado de la pérdida de la primera vocal de palabras como *nte ${ }^{1} \mathrm{t}^{\mathrm{h}} \mathrm{un}^{1}>/ \mathrm{nt}^{\mathrm{h}} \mathrm{o}^{2} / \rightarrow\left[\mathrm{n} \cdot \mathrm{t}^{\mathrm{h}} \mathrm{o}^{2}\right]$ 'nariz' (Kirk 1966; Wagner 2018).

7 Ver González (2021) para un análisis alternativo del mazateco de Chiquihuitlán, quien considera a la glotal como la primera consonante de una secuencia CC: $/ \mathrm{P}^{\mathrm{n}} \mathrm{d} /$. Mismo análisis que plantean Beal (2011) y Nakamoto (2020), para los mazatecos de Soyaltepec y Ayautla, respectivamente. 
Cuadro 9. Palabras con $/{ }^{\text {nn}} \mathbf{d} /$

\begin{tabular}{|c|c|}
\hline EJEMPLO & ZonA \\
\hline$/ \mathrm{fi}^{2} \mathrm{Pn} \mathrm{de}^{21 /} /$ 'liendre' & $\begin{array}{l}\text { Coyomeapan, Tlacotepec, Huehuetlán, Cuaunecuiltitla, } \\
\text { Ateixtlahuaca }\end{array}$ \\
\hline $\begin{array}{l}/{ }^{2 n} \mathrm{di}^{1} / \text { 'fiebre' } \\
/ \mathrm{ji}^{1} \mathrm{Pn}_{\mathrm{ne}} \mathrm{de}^{1 /} \text { 'adobe' }\end{array}$ & $\begin{array}{l}\text { Ocopetatillo, Eloxochitlán, Tecóatl, Acatepec, Yoloxochitlán, } \\
\text { Toxpalan, Los Cués, Zoquiapam }\end{array}$ \\
\hline /Pn $\mathrm{di}^{1} /$ 'estéril' & $\begin{array}{l}\text { Mazatlán, Tecomavaca, Chilchotla, Huautla, Asunción, } \\
\text { Huautepec, Tenango, Independencia, Tezonapa, Ayautla }\end{array}$ \\
\hline Pn $\mathrm{de}^{1 /}$ 'bochorno' & Chiquihuitlán \\
\hline
\end{tabular}

Con estas consonantes sobre-complejas damos por terminada la descripción de obstruyentes oclusivas y africadas (plosivas) en inicio silábico simple y damos paso a las fricativas.

\subsection{Fricativas}

Si dejamos atrás la mayor complejidad segmental del mazateco, es decir las plosivas, los otros sonidos obstruyentes de esta lengua son las fricativas. Sonidos que permiten el paso del flujo de aire por un canal estrecho en algún punto de la cavidad oral, cuya diferencia fundamental frente a las oclusivas es el rasgo [+continuo]. Todos estos segmentos son sordos en las lenguas mazatecas.

A continuación, se muestra la clase natural de las fricativas simples encontradas en las lenguas mazatecas, seguidos de ejemplos con la /s/. 
(16) Fricativas: clase natural [+consonántico, -resonante, + continuo] Lista de fonemas fricativos simples: / $\phi, \mathrm{s}, \mathrm{J} / \mathrm{s}, \mathrm{h} /$

Cuadro 10. Palabras con / s /

\begin{tabular}{cl}
\hline EJEMPLO: 'LUNA, MEs' & ZoNA \\
\hline$/ \mathrm{so}^{4} /$ & Coyomeapan, Tlacotepec, Huehuetlán, \\
& Cuaunecuiltitla, Ateixtlahuaca \\
$/ \mathrm{sa}^{4} /$ & Ocopetatillo, Eloxochitlán, Tecóatl, Acatepec, \\
$/ \mathrm{sa}^{3} /$ & Yoloxochitlán, Toxpalan, Los Cués, Zoquiapam, \\
& Mazatlán, Tecomavaca, Chilchotla, Huautla, \\
& Asunción, Huautepec, Tenango, Independencia, \\
& Jalapa, Teutila, Ixcatlán, Soyaltepec, Acatlán \\
& Ayautla, Chiquihuitlán \\
\hline
\end{tabular}

Las fricativas también permiten contrastes laríngeos, aunque en mucho menor medida que las oclusivas. La única fricativa posaspirada es la $/ \mathrm{s}^{\mathrm{h}} /$, presente en el mazateco del centro, Independencia y Ayautla; mientras que, en Ixcatlán, la /s/ y la / $/$ t tienen contrapartes glotalizadas (Wagner 2018).

\subsection{Resonantes: nasales, líquidas y aproximantes}

Los segmentos nasales pertenecen al grupo de fonemas resonantes. Las nasales se caracterizan por permitir la salida del aire por la nariz (con el descenso del velo) y contar con una constricción dentro del tracto bucal. Las nasales en las lenguas mazatecas pueden ser simples, 
aspiradas y glotalizadas. Por su sistematicidad y por simplificar la presentación, todos estos tipos se presentan en el siguiente cuadro.

Cuadro 11. Lista de fonemas nasales: [+resonante] [+nasal]

\begin{tabular}{|c|c|c|c|}
\hline \multirow[t]{2}{*}[+\text{nasal}]{} & \multirow[t]{2}{*}{ LaBIAL } & \multicolumn{2}{|c|}{ Coronal } \\
\hline & & {$[\operatorname{tant}]$} & {$[$-ant $]$} \\
\hline simple & $\mathrm{m}$ & $\mathrm{n}$ & $\mathrm{n}$ \\
\hline aspirada [+.g.e. $]$ & ${ }^{\mathrm{h}} \mathrm{m} \sim \mathrm{m}$ & ${ }^{\mathrm{h}} \mathrm{n} \sim \mathrm{n}$ & $\left({ }^{\mathrm{h}} \mathrm{n} \sim \mathrm{n}\right)$ \\
\hline glotalizada [+.g.c.] & ${ }^{2} \mathrm{~m} \sim \mathrm{m}$ & ${ }^{2} \mathrm{n} \sim \mathrm{n}$ & ${ }^{3} \mathrm{n} \sim \mathrm{\mu}$ \\
\hline
\end{tabular}

Antes de dar por hecho estos contrastes, conviene presentar aquí también al resto de fonemas resonantes de estas lenguas, pues el poder explicativo aumenta en la medida que se contempla en conjunto la clase natural de segmentos [+resonante, -silábico].

Las consonantes líquidas y semiconsonantes (o aproximantes) de las lenguas mazatecas son / 1, c (r), j, w /. Los sonidos líquidos se caracterizan por su gran sonoridad dentro del ámbito consonántico, aunque tengan aún algún contacto claro entre articuladores; mientras, los sonidos aproximantes que se pronuncian sin el desequilibrio de presiones de aire en el tracto bucal que puede provocar turbulencia. Así, la diferencia entre líquidas y aproximantes sería el rasgo [+/-consonántico]:

(17) Clases naturales resonantes: líquidas versus aproximantes Líquidas (laterales y vibrantes): [+resonante, -aproximante, +consonántico]

Aproximantes: [+resonante, +aproximante, -consonántico] 
A continuación, se muestran ejemplos de las resonantes coronales $/ \mathrm{n} / \mathrm{y} / 1 /$.

\section{Cuadro 12. Palabras con $/ \mathbf{n} /$}

\begin{tabular}{|c|c|}
\hline EJEMPLO: 'AÑO’ & ZoNa \\
\hline$/ \mathrm{nu}^{4} /$ & $\begin{array}{l}\text { Coyomeapan, Tlacotepec, Huehuetlán, Cuaunecuiltitla, Ateix- } \\
\text { tlahuaca, Mazatlán, Tecomavaca, Chiquihuitlán, Jalapa, Teutila, } \\
\text { Soyaltepec, Acatlán }\end{array}$ \\
\hline$/ \mathrm{no}^{4} /$ & $\begin{array}{l}\text { Ocopetatillo, Eloxochitlán, Tecóatl, Acatepec, Yoloxochitlán, } \\
\text { Toxpalan, Los Cués, Zoquiapam, Chilchotla, Huautla, Asunción, } \\
\text { Huautepec, Tenango, Ixcatlán }\end{array}$ \\
\hline$/ \mathrm{nu}^{4} \sim \mathrm{no}^{4} /$ & Independencia, Tezonapa \\
\hline$/ \mathrm{nu}^{2} /$ & Ayautla \\
\hline
\end{tabular}

Cuadro 13. Palabras con ///

\begin{tabular}{|c|c|}
\hline EJEMPLO & Zona \\
\hline$/ \mathrm{li}^{2} \mathrm{go}^{4} /$ 'oreja' & $\begin{array}{l}\text { Coyomeapan, Tlacotepec, Huehuetlán, Cuaunecuiltitla, } \\
\text { Ateixtlahuaca }\end{array}$ \\
\hline$/ \mathrm{lo}^{2} \mathrm{a}^{1 / /}$ 'naranja' & $\begin{array}{l}\text { Ocopetatillo, Eloxochitlán, Tecóatl, Acatepec, Yoloxochi- } \\
\text { tlán, Toxpalan, Los Cués, Zoquiapam }\end{array}$ \\
\hline$/ \mathrm{la}^{1} \mathrm{ha}^{1} /$ 'piedra' & Mazatlán, Tecomavaca \\
\hline$/ \mathrm{la}^{1} \mathrm{hao}^{1 /}$ 'piedra' & Chilchotla, Huautla, Asunción, Huautepec, Tenango \\
\hline$/ \mathrm{li}^{4} \mathrm{kie}^{4} /$ 'zopilote' & Independencia, Tezonapa \\
\hline$/ \mathrm{Pi}^{2} \mathrm{la}^{2} \mathrm{aa}^{1} /$ 'naranja' & Ayautla \\
\hline$/ \mathrm{li}^{22} \mathrm{ma}^{4} /$ 'gemelos' & Chiquihuitlán \\
\hline /li ${ }^{4} \mathrm{ku}^{1 /}$ 'loro' & Jalapa, Teutila \\
\hline$/ \mathrm{la}^{2} \mathrm{t}_{\mathrm{g}} \mathrm{o}^{1 /}$ 'Gelasio' & Ixcatlán \\
\hline$/ \mathrm{u}^{2} \mathrm{me}^{2} \mathrm{ta}^{2} /$ 'botella' & Soyaltepec, Acatlán \\
\hline
\end{tabular}


Una vez presentados los ejemplos de resonantes simples, retomamos la distribución de la complejidad laríngea mazateca. A diferencia de las obstruyentes, donde tenemos secuencias hC (en el caso de las "preaspiradas") y fonemas posaspirados, las resonantes reabren este debate, mostrando que resulta fundamental distinguir entre estas clases naturales. En este trabajo, las resonantes aspiradas se reanalizan de la siguiente manera:

(18) a. hC "pre-aspirado" segmento complejo = consonante aspirada. b. ${ }^{*} \mathrm{C}^{\mathrm{h}}$ "pos-aspirada" No contrastivo en Cs (discusión más adelante) (alofonía en Huautla y voz murmurada en Jalapa)

Cuando las resonantes portan un gesto aspirado al inicio se trata de consonantes complejas con la aspiración como rasgo secundario. Esto se alinea tipológicamente con la implementación de rasgos laríngeos en las resonantes, alineados al inicio o bien a lo largo de su producción, en consecuencia, con el principio de ligamiento de Kingston (1985) y la descripción de muchos sistemas lingüísticos (Ladefoged \& Maddieson 1996; Howe \& Pulleyblank 2001).

Sobre los casos con gestos laríngeos "pos-aspirados", dentro de las resonantes estos están ausentes a nivel fonológico en las lenguas y variantes mazatecas, distribución que apoya la propuesta de segmentos complejos resonantes (pre)aspirados (ver excepciones más abajo). Así, contaríamos en las lenguas mazatecas con consonantes resonantes preaspiradas. A continuación, presentamos los rasgos distintivos 
de esta clase segmental, la lista de fonemas y ejemplos con la nasal alveolar y la semiconsonante coronal.

(19) Resonante aspirada [+resonante, +glotis extendida] Lista de fonemas resonantes aspirados: $/{ }^{h} \mathrm{~m},{ }^{\mathrm{h}} \mathrm{n},{ }_{\mathrm{h}} \mathrm{n}, \mathrm{h}_{\mathrm{j}},{ }^{\mathrm{h}} \mathrm{w}^{8} /$

\section{Cuadro 14. Palabras con $/{ }^{\mathrm{h}} \mathrm{n} /$}

\begin{tabular}{ll}
\hline $\begin{array}{l}\text { EJEMPLO } \\
\text { 'MILPA' }\end{array}$ & Zona \\
\hline $\mathrm{h}_{\mathrm{nu}}{ }^{1 /}$ & $\begin{array}{l}\text { Coyomeapan, Tlacotepec, Huehuetlán, Cuaunecuiltitla, Ateixtlahuaca, } \\
\text { Mazatlán, Tecomavaca, Independencia, Tezonapa, Ayautla, Chiquihui- } \\
\text { tlán, Jalapa, Teutila, Soyaltepec, Acatlán }\end{array}$ \\
& $\begin{array}{l}\text { Ocopetatillo, Eloxochitlán, Tecóatl, Acatepec, Yoloxochitlán, Toxpa- } \\
\text { lan, Los Cués, Zoquiapam, Chilchotla, Huautla, Asunción, Huautepec, } \\
\text { Tenango, Ixcatlán }\end{array}$ \\
&
\end{tabular}

Cuadro 15. Palabras con $/{ }^{\mathbf{h}} \mathbf{j} /$

\begin{tabular}{ll}
\hline EJEMPLO & ZoNA \\
\hline $\mathrm{h}_{\mathrm{jo}}{ }^{2 /}$ 'quieto' & $\begin{array}{l}\text { Ocopetatillo, Eloxochitlán, Tecóatl, Acatepec, Yoloxochitlán, } \\
\text { Toxpalan, Los Cués, Zoquiapam }\end{array}$ \\
$\mathrm{h}_{\mathrm{jo}}{ }^{3 /}$ 'quieto' & Chilchotla, Huautla, Asunción, Huautepec, Tenango \\
$\mathrm{h}_{\mathrm{j} \tilde{1}^{2} / \text { 'ocho' }}$ & Chiquihuitlán \\
\hline
\end{tabular}

${ }^{8}$ La aproximante o semiconsonante labio-velar es poco frecuente en las lenguas mazatecas, presente solo en el mazateco del centro (Huautla, Tenango, Chilchotla) en palabras como $/ \mathrm{jo}^{2 \mathrm{~h}} \mathrm{wi}^{2} /\left(\rightarrow\left[\mathrm{jo}^{2 \mathrm{~h}} \beta \mathrm{i}^{2}\right]\right)$ 'nube'. 
Frente a estas resonantes (pre)aspiradas, existen casos excepcionales de posaspiración en resonantes, con ejemplos recopilados solo en dos variantes: Jalapa y Huautla. En el caso del mazateco de Jalapa, cuando hay una consonante resonante seguida de un gesto de aspiración, lo que tenemos claramente es una vocal murmurada, como se ha descrito en trabajos como Blankenship (2002). Al parecer es la única lengua mazateca con este rasgo contrastivo en el núcleo silábico, es decir, que este rasgo laríngeo es parte de la vocal y no de la consonante.

(20) Voz murmurada en el mazateco de Jalapa
a) $/ \mathrm{mex}^{2 / /}$ 'querer'
b) $/ \mathrm{nin}^{2} /$ 'mazorca'
c) $/$ nạ $^{1} /$ 'nueve'

Para el caso de la variante de Huautla, perteneciente al mazateco del centro, el caso resulta en principio más complicado, pues no hay una clara realización de voz murmurada en la vocal sino un gesto aspirado intermedio con variación entre una aspiración aislada, o rastros acústicos dentro de la consonante o al inicio de la vocal. Steriade (1994: 258), con base en el trabajo de Pike \& Pike (1947), hace referencia a este caso tan particular del mazateco del centro: "All the dialects of Mazateco-Popolocan I have encountered differ from Huautla in lacking a widespread and systematic contrast between pre- and postaspirated or pre- and posglottalized onsets".

Un hecho importante es que estos casos se restringen a las nasales $/ \mathrm{m} / \mathrm{y} / \mathrm{n} /$ (contrario a Jalapa cuya voz murmurada tiene mucho mayor 
distribución). Además, estas palabras son contadas. De los pocos ejemplos obtenidos, enlistamos las siguientes palabras.

(21) $\left[\mathrm{m}^{\mathrm{h}}\right]$ y $\left[\mathrm{n}^{\mathrm{h}}\right]$ en el mazateco del centro

$\left[\mathrm{m}^{\mathrm{h}} \mathrm{e}^{4}\right]$ 'lo cuelga'

$\left[\mathrm{n}^{\mathrm{h}} \mathrm{i}^{4}\right] \quad$ 'oscuro'

Lo marcado y poco frecuente de estos ejemplos, así como su restricción únicamente al mazateco del centro, nos hacen pensar en una excepción que requiere aún mayor investigación. La posibilidad de tomar este sonido como una secuencia de dos consonantes se rechaza dada la ausencia total en cualquier otra variante de un inicio con consonante más fricativa. A nivel de contrastes fonémicos y rasgos distintivos no hay posibilidad de pensar en un fonema nasal preaspirado y otro posaspirado $\left(/ \mathrm{h} \mathrm{m} / \mathrm{y} / \mathrm{h} n / v s . / \mathrm{m}^{\mathrm{h}} / \mathrm{y} / \mathrm{h} /\right)$. De hecho, en varias descripciones (Filio 2014; Wagner 2018) se enuncian simplemente como nasales murmuradas: /m, ṇ/.

Indagando en mayor medida al respecto, encontramos dos hechos fundamentales (Eloy García, comunicación personal, agosto de 2020) para postular en este trabajo que estas nasales "posaspiradas" son alófonos de sus contrapartes preaspiradas. En primer lugar, estas realizaciones no ocurren de forma homogénea en la zona centro, parecen centrarse en las variantes de Huautla y Tenango, y no ocurren, o solo en menor medida en Chilchotla, Asunción y Huautepec, lo que aumenta la excepcionalidad del caso. En segundo, lugar, los ejemplos mostrados en (20) tienen variación entre los hablantes en Huautla, que implementan 
la aspiración en diferentes momentos de la nasal (esta alofonía ya es presentada desde Pike \& Pike 1947: 80). Así, con base en esta variación inter e intra dialectal y la excepcionalidad léxica de casos posaspirados, proponemos la existencia de los fonemas $/{ }^{h} \mathrm{~m},{ }_{\mathrm{h}} /(\mathrm{o} / \mathrm{m}, \mathrm{n} /)$ con realizaciones pre y posaspiradas en variación libre: $\left[{ }^{\mathrm{h}} \mathrm{m},{ }^{\mathrm{h}} \mathrm{n} \sim \mathrm{m}, \mathrm{n} \sim\right.$ $\mathrm{m}^{\mathrm{h}}, \mathrm{n}^{\mathrm{h}}$, como se ilustra en (21), con los ejemplos ya mostrados en (20).

(22) Nasales aspiradas en el mazateco del centro ${ }^{9}$

$$
\begin{aligned}
& /{ }^{\mathrm{h}} \mathrm{me}^{4} / \rightarrow\left[{ }^{\mathrm{h}} \mathrm{me}^{4} \sim \mathrm{me}^{4} \sim \mathrm{m}^{\mathrm{h}} \mathrm{e}^{4}\right] \text { 'lo cuelga' } \\
& /{ }^{\mathrm{h}} \mathrm{ni}^{4} / \rightarrow\left[{ }^{\mathrm{h}} \mathrm{ni}^{4} \sim \mathrm{nii}^{4} \sim \mathrm{n}^{\mathrm{h}} \mathrm{i}^{4}\right] \quad \text { 'oscuro' }
\end{aligned}
$$

Con base en el análisis presentado, esquematizamos a continuación la nueva propuesta sobre la distribución del rasgo aspirado en las lenguas mazatecas para aclarar el estatus de segmentos complejos versus secuencias fonotácticas en estos sistemas.

Cuadro 16. Distribución del rasgo [+g.e.] (aspiración) en las lenguas mazatecas

\begin{tabular}{lll}
\hline$[+$ g.e. $]$ & "PRE-ASPIRADo" & "POS-ASPIRADo" \\
\hline Obstruyentes & $\mathrm{hC}(\mathrm{CC}=$ inicio complejo $)$ & $\mathrm{C}^{\mathrm{h}}(\mathrm{C}=$ segmento complejo $)$ \\
Resonantes & ${ }^{\mathrm{h}} \mathrm{C}(\mathrm{C}=$ segmento complejo $)$ & No contrastivo en $\mathrm{Cs}$ \\
& & $($ voz murmurada en Jalapa \\
& & y alofonía en Huautla-Tenango $)$
\end{tabular}

9 Otro caso es el de la palabra $\left[\mathrm{nh}^{\mathrm{h}}{ }^{4}\right]$ 'sangre', el cual parece tener menor variación que los otros items. Kirk (1966) mismo cita el ejemplo como consonante posaspirado en Huautla. Por ahora no tenemos mayor explicación al respecto más que tomar esta distribución defectiva como excepcional. 
En relación al otro rasgo laríngeo, [+glotis constreñida], presentamos ahora la distribución y análisis de la glotalización en las lenguas mazatecas dentro de las resonantes. Al igual que con la aspiración, para este tipo de segmentos tipológicamente esperamos que un rasgo secundario se ancle a la izquierda de una consonante resonante. Esto es lo que sucede en la agrupación mazateca, como mostramos en la siguiente lista, ilustrados con ejemplos de la $/{ }^{2} \mathrm{n} /$.

(23) Resonante glotalizada [+resonante, + glotis constreñida] Lista de fonemas resonantes glotalizados: / ${ }^{\mathrm{W}},{ }^{\mathrm{P}} \mathrm{m},{ }^{\mathrm{P}} \mathrm{n},{ }^{\mathrm{P}} \mathrm{n},{ }^{\mathrm{P}} \mathrm{j},{ }^{\mathrm{P}} 1 /$

Cuadro 17. Palabras con / 'n /

\begin{tabular}{|c|c|}
\hline EJEMPLO & Zona \\
\hline $\begin{array}{l}\text { / } \mathrm{Ju}^{2} \mathrm{nu}^{31 /} \text { 'hierba santa' } \\
/ \mathrm{no}^{23} \mathrm{ni}^{3} / \text { 'mala mujer' }\end{array}$ & $\begin{array}{l}\text { Coyomeapan, Tlacotepec, Huehuetlán, Cuaunecuiltitla, } \\
\text { Ateixtlahuaca }\end{array}$ \\
\hline $\begin{array}{l}/ \mathrm{fa}^{4} \mathrm{na}^{4} / \text { 'hierba santa' } \\
/ \mathrm{na}^{2} \mathrm{ni}^{4} / \text { 'mala mujer' } \\
/ \mathrm{na}^{1 /} \text { 'brillante' }\end{array}$ & $\begin{array}{l}\text { Ocopetatillo, Eloxochitlán, Tecóatl, Acatepec, Yoloxo- } \\
\text { chitlán, Toxpalan, Los Cués, Zoquiapam }\end{array}$ \\
\hline$/ \mathrm{na}^{2} \mathrm{ni}^{4} /$ 'mala mujer' & Chilchotla, Huautla, Asunción, Huatepec, Tenango \\
\hline /na ${ }^{1 /}$ 'brillante' & Independencia, Tezonapa, Ayautla, Ixcatlán \\
\hline /'ne²/ 'idioma' & Chiquihuitlán \\
\hline$/{ }^{2} a^{2} /$ 'brillante' & Jalapa, Teutila, Soyaltepec, Acatlán \\
\hline
\end{tabular}

En el caso de consonantes resonantes "pos-glotalizadas", como podrían aparentar en la escritura palabras como 'mecate', n'ó en la zona alta y $n$ 'ú en Jalapa, en realidad sucede lo mismo que con el caso de las obstruyentes, hablamos de un tipo de fonación en la vocal (voz 
laringizada) y no de un rasgo consonántico (ver discusión en García 2013: 93-96). Para la variante y lengua de Ixcatlán, no se propone la existencia de resonantes posglotalizadas a nivel fonémico (Wagner 2018: 50), hecho que consideramos una característica areal de la zona baja (salvo Jalapa). Así, con base en los trabajos antes citados y nuestros propios registros, el Cuadro 18 muestra la distribución de la glotalización en el sistema consonántico pan-mazateco, delimitando los casos de segmentos complejos, sin que para este rasgo se postule alguna secuencia consonántica.

Cuadro 18. Distribución del rasgo [+g.c.] (glotalización) en las lenguas mazatecas

\begin{tabular}{|c|c|c|}
\hline [+g.c. $]$ & “PRE-GLOTALIZADO” & "POS-GLOTALIZADO" \\
\hline Obstruyentes & No hay casos & $\begin{array}{l}\mathrm{C}^{?} / \underline{\mathrm{V}} \\
\left(\mathrm{C}^{?}-\mathrm{C} \text { glotalizada, tendencia zona baja y Ayautla }\right. \\
\mathrm{CV} \text { - rasgo vocálico, tendencia en zona alta y Jalapa) }\end{array}$ \\
\hline Resonantes & $\begin{array}{l}{ }^{2} \mathrm{C}(\mathrm{C}=\text { segmento } \\
\text { complejo })\end{array}$ & $\begin{array}{l}\text { CV (rasgo vocálico) } \\
\text { (zona alta, mazateco occidental y del centro) }\end{array}$ \\
\hline
\end{tabular}

Este recorrido por los diferentes tipos de segmentos mazatecos resultaba fundamental para el tema de la fonotáctica de estas lenguas. En particular, por el análisis de distintos gestos articulatorios como rasgos secundarios, tanto nasales como laríngeos, para conformar fonemas simples, complejos y sobre-complejos en estas lenguas. Lo anterior, a diferencia de un análisis de segmentos independientes que crearían diferentes tipos de secuencias consonánticas en estos sistemas (cf. Beal 2011; Nakamoto 2020). 
Con base en lo ilustrado hasta el momento, podemos formalizar la fonotáctica y sílaba CV mazateca a partir de las siguientes restricciones dentro de la Teoría de Optimidad (Prince \& Smolensky 1993; Kager 1999: 93).

(24) Inicio

* $[\sigma \mathrm{V}$ 'El inicio silábico es obligatorio'

(25) NoCoda

*C ] $\sigma$ 'Se prohíben segmentos en coda silábica'

(26) Plantilla silábica básica: CV

La restricción de INICIO evidencia la obligatoriedad de ataque consonántico en todas las sílabas de las lenguas mazatecas, mientras que la restricción NoCoda enuncia la prohibición de consonantes a final de sílaba. Ambas restricciones se observan sin excepciones en las lenguas mazatecas, es decir, que se encuentran altamente jerarquizadas en estas gramáticas.

Con estas conclusiones preliminares, damos pie a la sección de posibles secuencias consonánticas a principio de sílaba en las lenguas mazatecas, que mostrará la plantilla silábica posible CCV. 
4. INICIOS COMPLEJOS

4.1. CC: fricativa + plosiva

En las lenguas mazatecas es permitido el inicio complejo a nivel silábico; sin embargo, en palabras nativas se encuentra mayoritariamente restringido a la secuencia de fricativa + plosiva . Esta secuencia ocurre en todas las variantes, conformada por un segmento [+consonántico, + continuo], las fricativas $/ \mathrm{s}, \mathrm{s} / \int, \mathrm{h} /$, seguido de uno [+consonántico, -continuo], que agrupa oclusivas y africadas como la clase natural de plosivas: / t , $\mathrm{k}, \widehat{\mathrm{ts}}, \widehat{\mathrm{t}} \mathrm{T}, \widehat{\mathrm{tS}} /$. (Esta secuencia consonántica se describe desde el trabajo de Pike \& Pike 1947: 80).

Contrario a la restricción fonotáctica de lenguas como el español, que prohíbe que una palabra comience con el grupo consonántico formado por/s/ más una consonante, este inicio resulta parte esencial de la fonotáctica mazateca. Lo anterior debido a su alta frecuencia y distribución en el sistema, con lo que resulta una secuencia no marcada, que se alinea además a la tipología fonológica de muchas lenguas, permitiendo la consecución de segmentos [+continuo] seguido de [-continuo] (Carlisle \& Cutillas 2015 y referencias ahí citadas). En (27), se esquematiza esta descripción. 
(27) Inicio complejo mazateco: $\mathrm{CC}$ [fricativa + plosiva $]^{10}$
a. $\mathrm{C} 1=$ fricativa $/ \mathrm{s}, \mathrm{s} / \int, \mathrm{h} / \quad[+$ continua $]$
b. $\mathrm{C} 2=$ plosiva [-continua]
i. oclusiva / $\mathrm{t}, \mathrm{k} /$
(st, st/ft, ht; sk, sk/fk, hk)

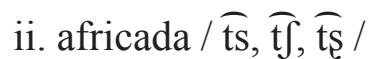
(sts, hts; $\sqrt{\mathrm{t} f}$, ht $\widehat{\mathrm{t}} ;$ h htş)
iii. prenasalizada / ${ }^{n} d,{ }^{n} \mathrm{~g},{ }^{n} \widehat{d},{ }^{n} \overline{d z} /$
$\left(h^{n} d, h^{n} g, h^{n} \widehat{d}, h^{n} \widehat{d z}\right.$.

Nótese que todas estas consonantes y secuencias son sordas, salvo las enlistadas en iii, donde el inicio complejo comienza con una fricativa glotal sorda, pero la segunda consonante es una prenasalizada. A continuación, mostramos algunos ejemplos de estas secuencias, incluyendo casos de fricativa más oclusiva, africada y prenasalizada con al menos una consonante coronal dentro de la secuencia.

10 Dentro de la literatura, la excepción a esta generalidad de inicios complejos de fricativa más oclusiva es la propuesta de Wagner (2018: 212) quien propone el segmento continuo como una consonante silábica, hecho que lleva al autor a concluir que no existen secuencias consonánticas en el mazateco de Ixcatlán (Wagner 2018: 69). Contrario a esta postura, consideramos en este trabajo que el análisis de una secuencia, y por ende un inicio complejo, es más económico descriptivamente hablando, además de lo aceptable que resulta la secuencia $\mathrm{SC}$ a nivel tipológico. Si bien, se reconoce que tanto en esta variante como en el mazateco de Zoquiapam existen nasales silábicas (Wagner 2018: cap. 5; Martínez 2020). 


\section{Cuadro 19. Palabras con / st / 11}

\begin{tabular}{|c|c|}
\hline EJEMPLO & Zona \\
\hline$/$ sti $^{3} \mathrm{na}^{3} /$ 'Justina' & $\begin{array}{l}\text { Ocopetatillo, Eloxochitlán, Tecóatl, Acatepec, Yoloxochi- } \\
\text { tlán, Toxpalan, Los Cués, Zoquiapam }\end{array}$ \\
\hline$/ \mathrm{tga}^{3} \cdot \mathrm{sta}^{2} /$ 'Constantino' & Chilchotla, Huautla, Asunción, Huautepec, Tenango \\
\hline$\widehat{t}_{\mathrm{fi}}{ }^{2} \mathrm{r}^{2} \cdot \mathrm{sto}^{2} /$ 'papera' & Independencia, Tezonapa \\
\hline$/ \mathrm{ste}^{2} \mathrm{ta}^{2 \mathrm{r}} \mathrm{a}^{2} /$ 'alcanza' & Chiquihuitlán \\
\hline /sta ${ }^{2} \mathrm{na}^{1} /$ 'lámina' & Jalapa, Teutila \\
\hline$/ \mathrm{Pi}^{2} \mathrm{sti}^{1} /$ 'aceite' & Ixcatlán $^{12}$ \\
\hline$/$ ni $^{2} \mathrm{sti}^{2} /$ 'muchacho' & Soyaltepec, Acatlán \\
\hline
\end{tabular}

\section{Cuadro 20. Palabras con / ht /}

\begin{tabular}{ll}
\hline EJEMPLO & ZoNA \\
\hline$/ h^{1}{ }^{1 /}$ 'voz' & $\begin{array}{l}\text { Coyomeapan, Tlacotepec, Huehuetlán, Cuaunecuiltitla, } \\
\text { Ateixtlahuaca } \\
\end{array}$ \\
$\begin{array}{l}\text { Ocopetatillo, Eloxochitlán, Tecóatl, Acatepec, Yoloxochi- } \\
\text { 'podrida (madera)' } / \mathrm{hti}^{3} /\end{array}$ & $\begin{array}{l}\text { tlán, Toxpalan, Los Cués, Zoquiapam } \\
\text { 'cortar' }\end{array}$ \\
$/ \mathrm{hti}^{1}$ ' 'se va a quemar' & \\
$/ \mathrm{hta}^{1 /}$ 'voz' & $\begin{array}{l}\text { Mazatlán, Tecomavaca, Chilchotla, Huautla, Asunción, } \\
\text { Huautepec, Tenango }\end{array}$ \\
\hline
\end{tabular}

11 Mazatlán no cuenta con secuencias /st/ porque la fricativa evolucionó a una aspiración en estos casos: $\mathrm{s}>\mathrm{h}$.

12 Para Wagner (2018) esta no es una secuencia, sino una consonante silábica seguida de una oclusiva heterosilábica. 
Cuadro 21. Palabras con / sts /

\begin{tabular}{ll}
\hline EJEMPLO & Zona \\
\hline$/$ stsi $^{3} /$ 'lluvia' & $\begin{array}{l}\text { Mazatlán, Tecomavaca, Independencia, Tezonapa, Ixca- } \\
\text { tlán, Soyaltepec, Acatlán }\end{array}$ \\
\hline
\end{tabular}

\section{Cuadro 22. Palabras con / hts /}

\begin{tabular}{|c|c|}
\hline EJEMPLO & ZONA \\
\hline /htse ${ }^{3}$ /'lluvia' & $\begin{array}{l}\text { Coyomeapan, Tlacotepec, Huehuetlán, Cuaunecuiltitla, } \\
\text { Ateixtlahuaca }\end{array}$ \\
\hline /htsi ${ }^{3} /$ 'lluvia' & $\begin{array}{l}\text { Ocopetatillo, Eloxochitlán, Tecóatl, Acatepec, Yoloxochi- } \\
\text { tlán, Toxpalan, Los Cués, Zoquiapam }\end{array}$ \\
\hline$/ \widehat{h t s i}^{3} /$ 'lluvia’ & Mazatlán, Tecomavaca \\
\hline$/ \widehat{h t s i}^{3} /$ 'lluvia' & Chilchotla, Huautla, Asunción, Huautepec, Tenango \\
\hline
\end{tabular}

\section{Cuadro 23. Palabras con / $h^{\mathrm{n}} d /$}

\begin{tabular}{ll}
\hline EJEMPLO & Zona \\
\hline$/ \mathrm{h}^{\mathrm{n}} \mathrm{da}^{2} /$ 'mal olor' & $\begin{array}{l}\text { Coyomeapan, Tlacotepec, Huehuetlán, Cuaunecuiltitla, } \\
\text { Ateixtlahuaca }\end{array}$ \\
$/ \mathrm{to}^{2} \mathrm{~h}^{\mathrm{n}} \mathrm{di}^{2} /$ 'huaxmole' & $\begin{array}{l}\text { Ocopetatillo, Eloxochitlán, Tecóatl, Acatepec, Yoloxochi- } \\
\text { tlán, Toxpalan, Los Cués, Zoquiapam }\end{array}$ \\
& Mazatlán, Tecomavaca \\
$/ \mathrm{h}^{\mathrm{n}} \mathrm{da}^{2} /$ 'glotón' & Ayautla \\
$/ \mathrm{h}^{\mathrm{n}} \mathrm{da}^{1} /$ 'voz' & Chilchotla, Huautla, Asunción, Huautepec, Tenango \\
$/ \mathrm{to}^{2} \mathrm{~h}^{\mathrm{n}} \mathrm{di}^{2} /$ 'huaxmole' & Independencia \\
$/ \mathrm{h}^{\mathrm{n}} \mathrm{da}^{22} \mathrm{a}^{2} /$ 'chimuelo' & \\
\hline
\end{tabular}


Cuadro 24. Palabras con / $\mathbf{h}^{\mathrm{n}} \widehat{\mathrm{d}} \mathrm{d} /$

\begin{tabular}{|c|c|}
\hline EJEMPLO & Zona \\
\hline /ngo ${ }^{1} \mathrm{~h}^{\mathrm{n}}{ }^{\mathrm{d}} \mathrm{i}^{1}{ }^{1 /}$ 'Teotitlán' & $\begin{array}{l}\text { Coyomeapan, Tlacotepec, Huehuetlán, Cuaunecuiltitla, } \\
\text { Ateixtlahuaca }\end{array}$ \\
\hline $\begin{array}{l}\text { /i }{ }^{1} \mathrm{~h}^{\mathrm{n}} \widehat{\mathrm{d}_{3}} \mathrm{i}^{1 /} \text { 'Teotitlán' } \\
/ \mathrm{i}^{1} \mathrm{~h}^{\mathrm{n}} \widehat{\mathrm{d}} \mathrm{a}^{1 /} \text { 'llano' }\end{array}$ & $\begin{array}{l}\text { Ocopetatillo, Eloxochitlán, Tecóatl, Acatepec, Yoloxochi- } \\
\text { tlán, Toxpalan, Los Cués, Zoquiapam }\end{array}$ \\
\hline$/ \mathrm{h}^{\mathrm{n}} \overline{\mathrm{d} z \mathrm{a}^{2}} /$ 'hay' & Mazatlán, Tecomavaca \\
\hline$/ \mathrm{h}^{\mathrm{n}} \widehat{\mathrm{d}} \mathrm{i}^{1} /$ 'Teotitlán' & Chilchotla, Huautla, Asunción, Huautepec, Tenango \\
\hline
\end{tabular}

Cuadro 25. Palabras con / han $\widehat{\mathbf{d} \mathbf{z}_{6}} /$

\begin{tabular}{ll}
\hline EJemPlo ‘salado' & Zona \\
\hline$/ \mathrm{h}^{\mathrm{n}} \widehat{\mathrm{dzo}}{ }^{2} /$ & $\begin{array}{l}\text { Coyomeapan, Tlacotepec, Huehuetlán, Cuaunecuiltitla, } \\
\text { Ateixtlahuaca }\end{array}$ \\
$/ \mathrm{h}^{\mathrm{n}} \widehat{\mathrm{dza}}{ }^{2} /$ & $\begin{array}{l}\text { Ocopetatillo, Eloxochitlán, Tecóatl, Acatepec, Yoloxochi- } \\
\text { tlán, Toxpalan, Los Cués, Zoquiapam }\end{array}$ \\
$/ \mathrm{h}^{\mathrm{n}} \widehat{\mathrm{dz}} /\left(\rightarrow\left[\mathrm{h}^{\mathrm{n}} \mathrm{za}^{2}\right]\right)$ & Chilchotla, Huautla, Asunción, Huautepec, Tenango \\
\hline
\end{tabular}

\subsection{CC: otras secuencias}

En algunas lenguas y variantes en particular, hemos recopilado otras secuencias consonánticas con vibrante: / $\mathrm{k}$, tr, ${ }^{\mathrm{n}} \mathrm{gr},{ }^{\mathrm{n}} \mathrm{d} \mathrm{s} /$; sin embargo, son ejemplos muy marcados que consideramos excepcionales. Otras secuencias con vibrante pueden encontrarse en préstamos no tan recientes, como /bie.rne/ 'viernes' y /kru/ 'cruz' (Zoquiapam), y varios más en palabras modernas (ver Chávez Peón 2018b para un análisis más detallado de estos casos). 


\section{Cuadro 26. Palabras con / $\mathbf{k} /$}

\begin{tabular}{ll}
\hline EJEMPLO & ZoNA \\
\hline$/ \mathrm{rki}_{\sim}^{21}$ / 'este, ese' & Eloxochitlán \\
$/ \mathrm{rku}^{1 /}$ 'su cabeza' & Chiquihuitlán \\
$/ \mathrm{rka}^{1} /$ 'Lucas' & Ixcatlán $^{13}$ \\
\hline
\end{tabular}

En otras variantes puede también aparecer en préstamos, como / mie $^{2} \mathbf{r k u e}^{2}$ / 'miércoles' en Zoquiapam vs. / mi $^{2} \mathbf{s k u e}^{2}$ / en Chilchotla.

Cuadro 27. Palabras escritas con /tr/

\begin{tabular}{ll}
\hline EJEMPLO & ZoNa \\
\hline$/ \mathrm{tru}^{2} \widehat{\mathrm{t}} \tilde{\mathrm{a}}^{2}$ 'tábano' & Chiquihuitlán \\
$/ \mathrm{tra}^{2} \mathrm{ku}^{2} /$ 'pelón' & Jalapa \\
\hline
\end{tabular}

Cuadro 28. Palabras con / ${ }^{\mathrm{g} g r} /$

\begin{tabular}{ll}
\hline EJEMPLO & Zona \\
\hline$\widetilde{\mathrm{t} f \mathrm{i}^{2} \cdot{ }^{\mathrm{g}} \mathrm{gri}^{2} / \text { 'iguana' }}$ & Chiquihuitlán \\
\hline
\end{tabular}

Cuadro 29. Palabras con / $\mathrm{n} d \mathrm{f} /$

\begin{tabular}{ll}
\hline EJEMPLo & Zona \\
\hline$\overparen{\mathrm{t} \int \mathrm{a}^{3} \cdot{ }^{\mathrm{n}} \mathrm{d} \mathrm{ri}^{2} / \text { ' } \text { 'grillo'14 }}$ & Mazatlán, Tecomavaca \\
\hline
\end{tabular}

13 Otra interpretación según Wagner (2018) es que la vibrante es múltiple y silábica: $/ \mathrm{r}^{2} \mathrm{ka}^{1} /$.

${ }^{14}$ Uno de los dictaminadores nos hizo notar que la palabra/tfa3.ndri2/ 'grillo' (Cuadro 29) es probablemente un préstamo del náhuatl chopili ‘chapulín’ y que la palabra /t fi2ngri2/ 'iguana' de Chiquihuitlán (Cuadro 28) guarda también cierto parecido. 
En línea con la excepcionalidad ya mencionada, el mazateco de Chiquihuitlán y de Ayautla cuentan con secuencias a inicio de palabra de fricativa + nasal: /sm, sn, $\int \mathrm{n}$ / (la primera solo en Chiquihuitlán), que, pese a su particularidad frente a las otras lenguas, conserva la sucesión de rasgos [+continuo] de la fricativa más [-continuo] de las nasales, alineándose así, al menos en cuanto a rasgos distintivos se refiere, a la prototípica secuencia de estas lenguas: fricativa + plosiva. (La secuencia de fricativa + nasal evolucionó a una nasal (pre)aspirada en la mayoría de las lenguas mazatecas, ver Chávez Peón, Wagner, Filio \& García 2021).

(28) Secuencia fricativa más nasal (mazateco de Chiquihuitlán y Ayautla) Chiquihuitlán Ayautla (Nakamoto 2020: 103)
a) $/ \mathrm{sm} /: / \mathrm{smi}^{2} /$ 'suelta'
b) $/ \mathrm{sn} /: \quad / \mathrm{sne}^{2} /$ 'tepejilote' $/ \mathrm{sne}^{1 /} \quad$ 'tepejilote'
c) $/ \mathrm{fn} /: \quad / \mathrm{nu}^{3} /$ 'ardilla' $/ \mathrm{tca}^{2} \mathrm{Snu}^{41 /}$ 'ardilla'

Al analizar el mazateco de Chiquihuitlán, González (2021) incluye en su tesis el ejemplo / $\widetilde{\mathrm{t}} \mathrm{u}^{4} \mathrm{rna}^{4} /$ 'el animal de ella' con la secuencia /rn/; no obstante, dicho ejemplo está dentro del nivel morfológico, fuera del alcance del presente trabajo.

El caso particular del mazateco de Chiquihuitlán a nivel de las secuencias consonánticas encontradas responde al hecho de ser particularmente diferente al resto de las lenguas mazatecas. Su aislamiento geográfico y continuo contacto con lenguas como el cuicateco y chinanteco han zanjado diferencias notables, creando 
una inteligibilidad muy baja con respecto al resto (ver Chávez Peón, Wagner, Filio \& García. 2021). Otro caso muy marcado con secuencias únicas y un desarrollo diacrónico particular es el mazateco de Jalapa (ver la descripción de estas secuencias de la Norma de escritura mazateca para más detalles).

En resumen, en esta sección hemos presentado los casos de inicios complejos en la sílaba de las lenguas mazatecas, esto es, las secuencias consonánticas permitidas, siendo el patrón no marcado y común a todos los sistemas la secuencia: fricativa + plosiva. Lo anterior infringe la restricción fonotáctica de (23).

(29) *Complex [Inicio]

*[б $\mathrm{\sigma C} \quad$ 'Se prohíben inicios complejos'

De manera puntual, una restricción observada en todas las variantes es la de no permitir secuencias de dos consonantes [-continuo] como se formaliza a continuación.

(30) *CC [-cont][-cont] (Chávez Peón 2018b)

'Se prohíben secuencias de segmentos [-continuo] en inicio complejo'

Esta restricción se observa también en las secuencias observadas de fricativa más nasal en los mazatecos de Chiquihuitlán y Ayautla, que junto con las secuencias de fricativa + plosiva conforman secuencias de segmentos $\mathrm{C}[+$ cont $] \mathrm{C}[$-cont $]$. 
De esta manera, aunque sus combinatorias sean restringidas, las lenguas mazatecas sí permiten los inicios complejos en su estructura silábica, pero de un máximo de dos consonantes (*CCC) (cf. Nakamoto 2020: 105).

Con esto damos por terminada y cubierta la fonotáctica de inicios complejos en todas las lenguas mazatecas, mostrando a detalle la distribución y tipología de sus secuencias consonánticas. Damos ahora paso a las conclusiones de este trabajo.

\section{Conclusiones}

En el presente trabajo hemos mostrado una amplia perspectiva de la fonotáctica de las lenguas y variantes mazatecas a nivel consonántico. El nivel vocálico requiere otro trabajo a detalle, pues estas lenguas permiten no solo núcleos simples, sino también diptongos, triptongos y diferentes rasgos contrastivos como la nasalidad, voz no modal y tonos, así como la interacción de estos contrastes.

Una vez presentado y propuesto el inventario segmental panmazateco y sus particularidades, nos enfocamos en unos de los grandes retos fonológicos que plantean estos sistemas: el análisis de los rasgos laríngeos (aspiración y glotalización). Gestos que permean toda su estructura y cuyo análisis y estatus determinan en gran medida las secuencias permitidas en la fonotáctica mazateca. Después de hacer un recuento de análisis previos sobre estos rasgos (Pike \& Pike 1947; Steriade 1994; Silverman, Blankenship, Kirk \& Ladefoged 1995; 
Golston \& Kehrein 1998, entre otros), se propone un análisis general para las lenguas mazatecas en el caso de la aspiración y un continuum dialectal descriptivo para el caso de la glotalización.

Para el caso de la aspiración, García, Chávez Peón \& Polian (2012) y García (2013) proponen que el gesto "pre-aspirado" de las consonantes se trata en realidad de una secuencia CC, mientras el gesto pos-aspirado, se trata en efecto de una consonante compleja. En el presente trabajo, sin embargo, hacemos una precisión mayor, al dividir las clases naturales de obstruyentes y resonantes; mientras la propuesta mencionada aplica para las obstruyentes, dentro de las resonantes, la preaspiración sí constituye un rasgo secundario alineada a la izquierda del segmento, mientras que el gesto de aspiración pos-resonántico es inexistente para la gran mayoría de las variantes, solo se encuentra en Jalapa y en Huautla. En Jalapa corresponde al rasgo vocálico de voz murmurada, mientras que en Huautla se trata de un caso de alofonía, como una posible realización de un único fonema aspirado: $/ h_{n} /(o / n /) \rightarrow\left[{ }^{h} n \sim n \sim n^{h}\right]$. Lo anterior se esquematiza de manera unificada y general en el Cuadro 30 y, como se mostró en el trabajo, da lugar a las secuencias consonánticas descritas.

En el caso de la glotalización la ausencia de obstruyentes preglotalizadas ubica el debate en la realización del rasgo [+g.c.] entre la consonante y la vocal. La diferencia analítica consiste en describir esta glotalización como parte de la vocal o de la consonante. Así, de manera generalizada se describe la existencia de voz laringizada para la zona alta y Jalapa (Silverman, Blankenship, Kirk \& Ladefoged 1995; Golston \& Kehrein 1998; García 2013), mientras que en la mayor 
Cuadro 30. Distribución del rasgo laríngeo [+glotis extendida] (aspiración) en las lenguas mazatecas

\begin{tabular}{|c|c|c|}
\hline [+g.e.] & “PRE-ASPIRADO" & “POS-ASPIRADO" \\
\hline \multirow[t]{4}{*}{ Obstruyentes } & $\mathrm{hC}(\mathrm{CC}=$ inicio complejo $)$ & $\mathrm{C}^{\mathrm{h}}(\mathrm{C}=$ segmento complejo $)$ \\
\hline & /ht/ & $/ \mathrm{t}^{\mathrm{h}} /$ \\
\hline & $/ \mathrm{hts} /$ & $/ \mathrm{ts}^{\mathrm{h}} /$ \\
\hline & $/ \mathrm{h}^{\mathrm{n}} \mathrm{d} /$ & $/{ }^{n} t^{h} /$ \\
\hline \multirow[t]{3}{*}{ Resonantes } & ${ }^{\mathrm{h}} \mathrm{C}(\mathrm{C}=$ segmento complejo $)$ & No contrastivo en Cs \\
\hline & $/ \mathrm{h}_{\mathrm{n}} /$ & $* / \mathrm{n}^{\mathrm{h}} /$ \\
\hline & $(\mathrm{o} / \mathrm{n} /)$ & {$\left[\mathrm{n}^{\mathrm{h}} \sim n^{\mathrm{n}} \sim \mathrm{n}^{\mathrm{h}}\right]$ (alofonía Huautla-Tenango) } \\
\hline
\end{tabular}

parte de la zona baja (Wagner 2018) y Ayautla (cf. Nakamoto 2020) reportamos consonantes glotalizadas. El alto grado de variación, sobre todo en la zona alta, invita a realizar estudios aún más detallados.

\section{Cuadro 31. Distribución del rasgo laríngeo [+glotis constreñida] (glotalización)} en las lenguas mazatecas

\begin{tabular}{|c|c|c|}
\hline$[+$ g.c. $]$ & “PRE-GLOTALIZADO" & “POS-GLOTALIZADO" \\
\hline Obstruyentes & No hay casos & $\begin{array}{l}\mathrm{C}^{2} / \underline{V} \\
\left(\mathrm{C}^{?}-\mathrm{C} \text { glotalizada, tendencia zona baja }\right. \\
\quad \text { y Ayautla } \\
\mathrm{CV} \text { - rasgo vocálico, tendencia en zona alta } \\
\quad \text { y Jalapa })\end{array}$ \\
\hline Resonantes & $\begin{array}{l}{ }^{2} \mathrm{C} \\
\text { (segmento complejo) }\end{array}$ & $\begin{array}{l}\text { CV (rasgo vocálico) } \\
\text { (zona alta: mazateco de Puebla, noroeste, occi- } \\
\text { dental y del centro) }\end{array}$ \\
\hline
\end{tabular}


Una vez establecida la distribución de los rasgos laríngeos, en la sección de inicio complejo se detallaron los casos de secuencias permitidas en las lenguas mazatecas, en esencia fricativa más plosiva. Como $\mathrm{C} 1$, encontramos a las fricativas: $/ \mathrm{s}, \mathrm{J} / \mathrm{s}, \mathrm{h} /$, todos segmentos [+continuo]; como $\mathrm{C} 2$, esta posición la pueden ocupar una amplia gama de la clase de plosivas (segmentos [-continuo]): oclusivas simples, africadas y prenasalizadas. Repetimos a continuación el siguiente esquema que resume esta descripción.

(31) Inicio complejo mazateco: CC [fricativa + plosiva]
a. $\mathrm{C} 1=$ fricativa $/ \mathrm{s}, \mathrm{J} / \mathrm{s}, \mathrm{h} / \quad[+$ continua $]$
b. $\mathrm{C} 2=$ plosiva
[-continua]
i. oclusiva $/ \mathrm{t}, \mathrm{k} /$
(st, st/ft, ht; sk, sk/fk, hk)
ii. africada $/ \widehat{\mathrm{ts}}, \widehat{\mathrm{t} f}, \widehat{\mathrm{ts}} /$
(sts, hts; $\widetilde{\jmath \mathrm{t} f}$, ht $\widehat{h} ;$ hts $)$
iii. prenasalizada $/{ }^{\mathrm{n}} \mathrm{d},{ }^{\mathrm{n}} \mathrm{g},{ }^{\mathrm{n}} \widehat{\mathrm{d}},{ }^{\mathrm{n}} \widehat{\mathrm{dz}} /$
$\left(h^{n} d, h^{n} g ; h^{n} \widehat{d z}, h^{n} \widehat{d z}\right)$

Como ya hemos mencionado, el mazateco de Chiquihuitlán y el de Ayautla son particulares en varios aspectos, sobre las secuencias CC en particular, además de fricativa + plosiva, son las únicas variantes que conservan también la secuencias de fricativa + nasal. Aun con esta excepcionalidad, se alínea a la generalidad de las lenguas mazatecas en permitir solo secuencias de $\mathrm{C}[+$ continua $] \mathrm{C}[-$ continua $]$. Estas dos lenguas representan zonas muy marcadas dialectalmente hablando por su aislamiento del resto (Chávez Peón, Wagner, Filio \& García 2021), hecho que explica varias diferencias y excepciones en sus fonologías 
comparadas con el resto de las lenguas mazatecas (ver González 2021 y Nakamoto 2020 para más detalles y análisis alternativos). ${ }^{15}$

En términos de estructura silábica, con base en la sección de inicios simples de este trabajo definimos que es obligatoria la presencia de una consonante inicial en cada sílaba y que en mazateco no se permiten segmentos en coda. Sumado a lo anterior, y con base en la descripción de los casos de inicio complejo, hemos descrito también que las lenguas mazatecas sí permiten una secuencia de dos consonantes, en contra de la restricción *Complex; sin embargo, estas consonantes tienen una notable restricción dado que en palabras nativas únicamente se admiten secuencias de fricativa más plosiva (o más ampliamente, segmentos [-continuo] como $\mathrm{C} 2$ si incluimos las nasales de Chiquihuitlán y Ayautla). De manera marcada y minoritaria, en algunas variantes se permiten otras secuencias que contienen una vibrante, incluyendo léxico en particular (eclesiástico) y préstamos recientes (Chávez Peón 2018b), pero en ningún caso se admiten secuencias de dos consonantes [-continuo].

En conclusión, la fonotáctica de las consonantes de las lenguas mazatecas aquí descrita se resume y formaliza de manera esquemática con las siguientes restricciones y platilla.

15 Como se mencionó anteriormente, Nakamoto (2020: 105-106) reporta en la fonotáctica del mazateco de Ayautla secuencias triconsonánticas conformadas de una sibilante más plosiva más consonante glotal, así como una secuencia cuadriconsonántica: sibilante-plosiva-glotal-j. En el presente trabajo, dichas glotales se toman como rasgos secundarios de la consonante, es decir, como una obstruyente posglotalizada. 
(32) Inicio

‘El inicio silábico es obligatorio' $\quad(* \mathrm{~V}, * \mathrm{VC})$

(33) NoCodA

$\left({ }^{*} \mathrm{CVC}\right)$

'Se prohíben segmentos en coda silábica'

(34) *Complex [Inicio]

(Infringido en las lenguas mazatecas)

'Se prohíben inicios complejos'

(35) $* \mathrm{CC}[$-cont $][$-cont $]$

'Se prohíben secuencias de segmentos [-continuo] en inicio complejo'

(36) Plantilla silábica de las lenguas mazatecas: (C)CV(VV)

$\mathrm{CV}$ $*[\sigma \mathrm{V}$

$* \mathrm{~V},{ }^{*} \mathrm{VC}$

${ }^{*} \mathrm{CVC}$

$\left.{ }^{*} \mathrm{C}\right] \sigma$

$\mathrm{CCV}$

*CCCV

Todo lo anterior cubre la fonotáctica de las lenguas y variantes mazatecas a nivel consonántico de manera global y con un análisis unificado. Asimismo, ofrecimos una detallada propuesta de las articulaciones secundarias de estos sistemas con base en lineamientos tipológicos y la universalidad argumentativa de la teoría de rasgos distintivos y de geometría de rasgos. 
Descripciones anteriores, así como el trabajo de la Norma de escritura mazateca y la dialectología del Proyecto Nanginá han permitido ofrecer aquí una amplia visión de todos los sistemas fonológicos de la agrupación lingüística mazateca. Sin duda, este análisis podrá perfeccionarse con estudios posteriores, pero confiamos también que en su estado actual contribuya al estudio de las lenguas mazatecas a nivel teórico, descriptivo y aplicado.

\section{Agradecimientos}

Agradecemos sinceramente la invitación a este volumen y seguimiento al presente trabajo por parte de Mario Hernández Luna, coordinador de este Dossier sobre Temas de fonología otomangue (Cuadernos de Lingüística, El Colegio de México), así como los detallados comentarios de Carlos Wagner y a los dictaminadores de este artículo. Máté-

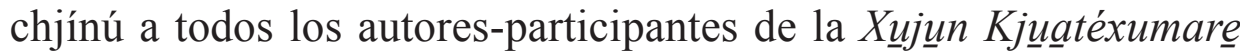
Énná (Norma de escritura del mazateco) de donde se citan varios de los ejemplos aquí presentados.

Agradecemos también al Instituto Nacional de Lenguas Indígenas (INALI), en particular a Rosa María Rojas, Juan Gregorio Regino, Nicandro González y Aileen Martínez, así como al Centro de Investigaciones y Estudios en Antropología Social (CIESAS) por su apoyo al Proyecto Nanginá de Documentación Lingüística y Dialectología Mazateca. 
REFERENCIAS

Agee, Daniel. 1993. Modal clitics in San Jerónimo Mazatec. SIL-México Workpapers 10. 1-28. https://mexico.sil.org/es/resources/ archives/10888.

Beal, Heather D. 2011. The segments and tones of Soyaltepec Mazatec (Vol. 53). Arlington: University of Texas /SIL International.

Blankenship, Barbara. 2002. The timing of nonmodal phonation in vowels. Journal of phonetics, 30(2). 163-191. DoI: 10.1006/ jpho.2001.0155.

Blevins, Juliette. 1995. The syllable in phonological theory. En Goldsmith, John A. (ed.), The handbook of phonological theory, 20644. (Blackwell Handbooks in Linguistics 1). Londres: Blackwell Publishers.

Blevins, Juliette. 2003. The independent nature of phonotactic constraints: An alternative to syllable-based approaches. En Féry, Caroline \& van de Vijver, Ruben (eds.), The syllable in optimality theory, 375-403. Cambridge: Cambridge University Press.

Bull, Brian E. 1984. Aspect formation of San Jerónimo Mazatec verbs, 93-117. (Workpapers 6). México: ILV.

Carlisle, Robert S. \& Cutillas Espinosa, Antonio. 2015. The Production of $/ \mathrm{sC}$ / onsets in a markedness relationship: Investigating the ontogeny phylogeny model with longitudinal data. En Yavas, Mehemet (ed.), 183-205. Unusual productions in phonology: Universal and language. Nueva York: Psychology press. 
Carrera Guerrero, Hugo. 2012. Patrones silábicos del mazateco de San Lorenzo Cuaunecuiltitla (Ponencia presentada en el Coloquio de Lingüística de la enah. Oaxaca, México).

Carrera Guerrero, Hugo. 2014. Fonología del mazateco de San Lorenzo Cuaunecuiltitla. Puebla: Benemérita Universidad Autónoma de Puebla. (Tesis de licenciatura).

Chávez Peón, Mario E. 2010. The interaction of metrical structure: tone and phonation types in Quiavini Zapotec. Vancouver: University of British Columbia. (Tesis doctoral).

Chávez Peón, Mario E. 2018a. Proyecto Nanginá 'nuestra tierra'. Documentación lingüística y dialectología del mazateco: metodología y resultados. En Montaño, Cynthia; Arellano, Inocencia \& Sánchez, Luis Alberto (coords.), ¿Qué hacer para registrar y preservar una lengua? Aproximaciones a la documentación lingüistica, 215-250. Querétaro, México: Universidad Autónoma de Querétaro.

Chávez Peón, Mario E. 2018b. Fonología de préstamos en el mazateco: adaptaciones fonotácticas. en Herrera, Esther. (ed.), Fonología segmental: procesos e interacciones, 115-147. México: El Colegio de México.

Chávez Peón, Mario E. \& Hernández, Fidel. 2021. Las lenguas tonales en México. En Chávez Peón, Mario E. \& de León, Lourdes (eds.), Lenguas mesoamericanas en el siglo XXI: enfoques socioculturales y tipológicos. (Colección México). México: ciesas.

Chávez Peón, Mario E.; Wagner, Carlos; Filio, Israel \& García, Eloy. 2021. Las lenguas mazatecas y sus variantes. En Cardona, Pedro 
(ed.), Lingüistica Amerindia. Querétaro: Universidad Autónoma de Querétaro.

Clements, G. Nick \& Keyser, Samuel J. 1983. CV Phonology: A generative theory of the syllable (Linguistic Inquiry Monograph 9). Cambridge, MA: MIT Press.

Clements, G. N., \& Hume, E. (1995). The internal organization of segments. En Goldsmith J. (ed.), Handbook of phonological theory. Cambridge, MA: Blackwell.

Clements, G. Nick. 1985. The geometry of phonological features, Phonology yearbook 2. 225-252. Cambridge: Cambridge University Press.

Clements, Nick G. 1999. Affricates as noncontoured stops. En Fujimura, Osamu; Brian, Joseph \& Palek, Bohumil (eds.), Proceedings of LP '98: Item order in language and speech. 271-299 Praga: The Karolinum Press. .

Filio García, Israel. 2011. La lengua ién Najndiaá. En Pérez López, María Soledad \& Cruz García, Fanny. Las lenguas originarias de México y sus alfabetos. México: Universidad Pedagógica Nacional. Filio García, Israel. 2014. Estructura fónica y acercamiento gramatical del Mazateco de Najndiá. México: Centro de Investigaciones y Estudios Superiores en Antropología Social. (Tesis de maestría). García García, Eloy. 2013. Fonología segmental y sistema tonal del mazateco de Río Santiago, Huautla. México: Centro de Investigaciones y Estudios Superiores en Antropología Social. (Tesis de maestría). 
García, Eloy; Chávez Peón, Mario E. \& Polian, Gilles. 2012. Contrastes laríngeos en el mazateco de Río Santiago (Huautla). (Ponencia presentada en el Coloquio sobre lenguas otomangues y vecinas, Biblioteca de Investigación Juan de Córdova, 20-22 de abril de 2012, Oaxaca, México).

Goldsmith, John A. 1976. Autosegmental phonology. Cambridge: Massachusetts Institute of Technology. (Tesis doctoral).

Golston, Chris, \& Kehrein, Wolfgang. 1998. Mazatec onsets and nuclei. International Journal of American Linguistics 64(4). 311337.

González Valseca, Carlos Misael. 2021. Fonología del mazateco de Chiquihuitlán de Benito Juárez, Oaxaca. México: Escuela Nacional de Antropología e Historia. (Tesis de licenciatura).

Gregorio, Juan. 1993. Alfabeto mazateco. Oaxaca, México: Instituto Oaxaqueño de las Culturas.

Gudschinsky, Sara C. 1956. Proto-Mazatec structure. Pennsylvania: University of Pennsylvania. (Tesis de maestría).

Gudschinsky, Sara C. 1958. Mazatec dialect history: A study in miniature. Language 34(4). 469-481.

Herrera, Esther. 2003. Tono, VOT y sonoridad en el mazateco de Santa Clara, Oaxaca. En Herrera Esther. \& Martín Pedro. (eds.), La tonía: dimensiones fonéticas y fonológicas. México: El Colegio de México.

Howe, Daring M. \& Pulleyblank, Douglas G. 2001. Patterns and timing of glottalisation. Phonology 18(45-80). 
Jamieson, Allan R. 1977. Chiquihuitlan Mazatec tone. Dallas, Texas: Summer Institute of Linguistics.

Jakobson, Roman; Fant, Gunnar \& Halle, Morris. 1952. Preliminaries to speech analysis. The distinctive features and their correlates. Boston: M.I.T. Press.Kager, René. 1999. Optimality theory. Cambridge: University Press.

Kehrein, Wolfgang \& Golston, Chris. 2004. A prosodic theory of laryngeal contrasts. Phonology 21(3). 325-357.

Kingston, John. 1985. The phonetics and phonology of the timing of oral and glotal events. Berkeley: Universidad de California. (Tesis doctoral).

Kirk, Paul L. 1966. Proto-Mazatec phonology. Seattle: University of Washington. (Tesis doctoral).

Kirk, Paul L. 1970. Dialect intelligibility testing: The Mazatec Study. International journal of American linguistics 36(3). 205-201.

Klint, Ryan David \& Filio García, Israel. 2018. 'Ien Nájndịa, el mazateco de Mazatlán Villa de Flores. En Marlett, Stephen A. (ed.), Ilustraciones fonéticas de lenguas amerindias. Lima: SIL International y Universidad Ricardo Palma. www.lengamer.org/publicaciones/ trabajos/mazateco_de_Mazatlan_afi.pdf

Ladefoged, Peter \& Maddieson, Ian. 1996. The sounds of the world's languages. Oxford, UK \& Cambridge, MA: Blackwell.

Lamiman, Jon. 2013. Ixcatlan Mazatec phonology. Ms.

Léonard, Jean Léo; dell'Aquila, Vittorio \& Gaillard-Corvaglia, Antonella. 2012. The Almaz (Atlas Lingüístico Mazateco): From 
geolinguistica data procesing to typological traits. Sprachtypologie und universalienforschung 65(1). 78-94.

Léonard, Jean Léo \& Fulcrand, Julien. 2016. Tonal inflection and dialectal variation in Mazatec. En Palancar, Enrique L. \& Léonard, Jean Léo (eds.), Tone and inflection, 163-167. News facts and new perspectives. Berlín: Mouton de Gruyter.

Léonard, Jean Léo; Heinsalu, Els; Patriarca, Marco; Sharma, Kiran \& Chakraborti, Anirban. 2017. Patterns of linguistic diffusion in space and time: The case of Mazatec. En Abergel F. et al. (eds.), Econophysics and sociophysics: Recent progress and future directions. New Economic Windows. Springer, Cham. https://doi. org/10.1007/978-3-319-477052-3_17

Ly Van Tu, Jean-Cyrille. 2015. Complexité structurale en mazatec et phonologie de laboratoire. Ms. Journée d'études du 5 mars. https:// www.academia.edu/11576126/Complexit\%C3\%A9_structurale_ en_mazatec_et_phonologie_de_laboratoire

Marlett, Stephen A. 1992. Nasalization in Mixtec languages. International journal of American linguistics 58. 425-435.

Martínez Carrera, Gloria. 2020. Kuiss 'ín maxkọaya én Ndáxa 'bì k'enga sindà én, meé xá ri 'niá. Morfología verbal del mazateco de San Juan La Unión, Zoquiapam: sistema de TAM y clase flexivas. México: Centro de Investigaciones y Estudios Superiores en Antropología Social. (Tesis de maestría).

Mehmet, Yavaş. 2006. Sonority and the acquisition of \#sC clusters. Journal of Multilingual Communication Disorders 4(3). 159-168. 
Morelli, Frida. 1999. The phonotactics and phonology of obstruent clusters in optimality theory. Maryland: University of Maryland at College Park. (Tesis doctoral).

Nakamoto, Shun. 2020. Tonología mazateca: San Bartolomé Ayautla. México. Universidad Nacional Autónoma de México. (Tesis de maestría).

Pike, Eunice V. 1956. Tonally diferentiated allomorphs in Soyaltepec Mazatec. Chicago: The University of Chicago Press.

Pike, Kenneth L. \& Pike, Eunice Victoria. 1947. Immediate constituents of Mazateco syllables. International Journal of American Linguistics 13(2). 78-91.

Prince, Alan \& Smolensky, Paul. 1993. Optimality theory: Constraint interaction in generative grammar, Rutgers Technical Reports TR-2 (véase también ROA 537). [2004, Blackwell Publishers.]

Silverman, Daniel; Blankenship, Barbara; Kirk, Paul \& Ladefoged, Peter. 1995. Phonetic structures in Jalapa Mazatec (Vol. Vol. 37, No. 1). Los Angeles: University of California.

Steriade, Donca. 1994. Complex onsets as single segments: The Mazateco pattern. En Cole, Jennifer \& Kisseberth, Charles (eds.), Perspectives in phonology (Lecture Notes 51), 203-291. Stanford: Center for the Study of Language and Information.

Uchihara, Hiroto. 2021. Clusters vs. units in Otomanguean: the case of Tlapanec (Mè'phàà) and Zapotec (Dixsa:). Cuadernos de Lingüistica de El Colegio de México 8, e224. Dor: 10.24201/ clecm. v8i0.224. 
Vielma Hernández, Jonathan Daniel. 2017. Panorama de los estudios lingüísticos sobre el mazateco. Cuadernos de Lingüística de El Colegio de México 4(1). 211-272.

Wagner Oviedo, Carlos de Jesús. 2017. Génesis de contrastes de estado glótico en oclusivas de las lenguas mazatecas. Manuscrito. https://www.academia.edu/35120796/G\%C3\%A9nesis_de_contrastes_de_estado_gl\%C3\%B3tico_en_oclusivas_de_las_lenguas_ mazatecas.

Wagner Oviedo, Carlos de Jesús. 2018. Xi kó ts'en fañe 'én Ningotsie: fonología segmental y tonal del mazateco de Ixcatlán. México: Escuela Nacional de Antropología e Historia. (Tesis de licenciatura).

Wagner Oviedo, Carlos de Jesús. 2021. Desarrollo diacrónico y distribución geográfica de las vocales en las lenguas mazatecas. Las lenguas mazatecas y sus variantes. En Cardona, Pedro (ed.), Lingüistica amerindia. Querétaro: Universidad Autónoma de Querétaro.

Wagner Oviedo, Carlos de Jesús; Manzano; Miriam \& Chávez Peón, Mario E. 2017. Cambio vocálico en el mazateco de Puebla y del Noroeste. (Ponencia presentada en el Congreso Internacional de Estudios sobre Lenguas Indígenas (CIELI) en la Facultad de Filosofía, Universidad Autónoma de Querétaro, 15-17 noviembre.) Xújun Kjụatéxumare Énná. Norma de escritura del mazateco. 2021. Filio García, Israel; García García, Eloy \& Chávez Peón, Mario E., en colaboración con todos los participantes (eds.). México: Instituto Nacional de Lenguas Indígenas. 
Zec, Draga. 2007. The syllable. En de Lacy, Paul (ed.), The Cambridge handbook of phonology. Cambridge: Cambridge University Press. 